\title{
Antisymmetrized Molecular Dynamics with Bare Nuclear Interactions: Brueckner-AMD, and Its Applications to Light Nuclei
}

\author{
Tomoaki Togashi ${ }^{1}$ and Kiyoshi Katō ${ }^{2}$ \\ ${ }^{1}$ Faculty of Advanced Life Science, Hokkaido University \\ ${ }^{2}$ Division of Physics, Graduate School of Science, Hokkaido University \\ Japan
}

\section{Introduction}

$A b$ initio calculation starting from the bare nuclear interactions is one of recent major theoretical subjects in nuclear physics. One hopes to understand the property of nuclei based on the nuclear forces against the background of the progress of the fundamental understanding of bare nuclear interactions and the study of unstable nuclei far from the beta stable line, which are expected to have structures different from stable nuclei. Nowadays, one can obtain the three-, four-, or few-body wave functions starting from the bare Hamiltonian by applying many kinds of few-body exact methods (Kamada et al., 2001). It is difficult to apply such methods directly to heavier nuclei in viewpoint of computational costs at this stage, therefore, in many of ab initio approaches, the effective interaction is constructed based on the bare interaction by applying, for example, the unitary correlated operator method (UCOM) (Neff \& Feldmeier, 2004) and so on. As one of remarkable achievements in ab initio approaches, the quantum Monte Carlo method (Wiringa et al., 2000) has presented the appearance of the two-alpha $\left({ }^{4} \mathrm{He}\right)$ cluster structure in ${ }^{8} \mathrm{Be}$ starting from the bare interactions. That suggests that one has the ability to discuss cluster structures based on the bare nuclear forces.

The existence of cluster states appearing around thresholds, which are weakly bound systems of subunits consisting of several nucleons to be strongly correlated each other, is predicted by the Ikeda diagram and the cluster models (Ikeda et al., 1972). The cluster states in some excited states of a light nucleus can be described as the states different from the mean-field-like structures. Cluster models in nuclear physics are able to describe these states successfully. In unstable nuclei, it is expected that mean-field-like and cluster-like structures coexist because their ground states often exist around the thresholds of decaying particles. The antisymmetrized molecular dynamics (AMD) (Kanada-En'yo et al., 1995) to be one of the recent developments of the cluster model has succeeded in understanding and predicting many properties of unstable nuclei. AMD can describe the nuclear structures without any assumption of configurations as the results of the energy variation in quantum mechanics. However, it is difficult for the bare nuclear interactions to be applied straightforwardly to the AMD framework because of the singularity and complexity in the interactions. Hence, AMD calculations have been performed with phenomenological interactions represented by 
the simple forms and adjusted parameters to be suitable for the wave functions of AMD so far.

For the purpose of the fundamental understanding of nuclear structures, we have recently developed a new AMD framework, “Brueckner-AMD” (Togashi \& Katō, 2007; Togashi et al., 2009), which makes AMD available to us with bare nuclear interactions. It is the basic idea of Brueckner-AMD that the effective interaction with no singularity to be applicable to the AMD wave functions is constructed starting from the bare interaction. Instead of the unitary transformation of the bare Hamiltonian, we introduce the effective interaction, G-matrix, based on the Brueckner theory to be combined with the single-particle orbits and energies solved by the AMD+Hartree-Fock (AMD-HF) method (Doté et al., 1997). In this framework, the G-matrix and single-particle states of AMD can be solved self-consistently so as to reflect structural changes of nucleus to the effective interactions. In that sense, Brueckner-AMD is a kind of ab initio calculations.

The appearance of alpha clusters has been studied in many light nuclei for many years. It is well known that the alpha particle, ${ }^{4} \mathrm{He}$, which is the unit of alpha clusters, has the strong stability as its threshold energy for a nucleon is about $20 \mathrm{MeV}$, while normal nuclei have about $8 \mathrm{MeV}$. Theoretical few-body studies (Kamada et al., 2001) have proven that the tensor force in the bare nuclear interaction, which has the operator: $S_{12}=3\left(\sigma_{1} \cdot \hat{r}\right)\left(\sigma_{2} \cdot \hat{r}\right)-\sigma_{1} \cdot \sigma_{2}$ where $\sigma_{1,2}$ represent the Pauli matrices, contributes more than half of the binding potential energy of the alpha particle, and the correlations induced by the tensor force play an important role in the structure of ${ }^{4} \mathrm{He}$ (Myo et al., 2009). Therefore, it is considered that the peculiar stability of the alpha particle supported by the tensor force contributions induces the strong correlations to realize the alpha clusters. However, the reason for the stability of alpha clusters in a nucleus has not yet been clarified, and it is still one of the central problems in nuclear theories at present. Although the recent $a b$ initio approach (Wiringa et al., 2000) has provided the result of the alpha-alpha cluster structure in ${ }^{8} \mathrm{Be}$ as previous mentioned, it has been difficult to explain the reason why the alpha-alpha cluster in ${ }^{8} \mathrm{Be}$ appears for the complicated wave function solved from the bare Hamiltonian. Therefore, as our works, we present the prescription to simulate and visualize the development of alpha-alpha clustering in ${ }^{8} \mathrm{Be}$, and elucidate its mechanism of the clustering as the result of effects of nuclear interactions in the Brueckner-AMD framework.

In this chapter, we plan to introduce the formulation and applications of Brueckner-AMD. In the second section, we present the formulation of Brueckner-AMD and focus on the details of the way how to solve the G-matrix in this framework. In the third section, we show the applications to several light nuclei and the study of alpha-alpha clustering in ${ }^{8} \mathrm{Be}$ in Brueckner-AMD. Finally, in the forth section, we conclude our works in this chapter.

\section{Formulation}

In this section, we explain the formulation of Brueckner-AMD. In the first subsection 2.1, we explain the concept of Brueckner-AMD, in which the G-matrix within the AMD framework can be calculated straightforwardly. In the second subsection 2.2, the detail of the way how to solve the G-matrix in Brueckner-AMD and its explicit examples are presented, and then the energy variation method in Brueckner-AMD is explained in the third subsection 2.3. 


\subsection{G-matrix in Brueckner-AMD}

In this framework, we use the $A$-nucleons wave function of AMD, which represents the Slater determinant of Gaussian wave packets to be satisfied with the Fermi statistics in quantum mechanics as

$$
|\Phi\rangle=\frac{1}{\sqrt{A !}} \operatorname{det}\left\{\left|\vec{Z}_{i}\right\rangle \cdot\left|\chi_{i}\right\rangle\right\},\left\langle\vec{r} \mid \vec{Z}_{i}\right\rangle=\left(\frac{2 v}{\pi}\right)^{3 / 4} \exp \left[-v\left(\vec{r}-\frac{\vec{Z}_{i}}{\sqrt{v}}\right)^{2}+\frac{\vec{Z}_{i}^{2}}{2}\right],
$$

where $\left|\vec{Z}_{i}\right\rangle$ and $\left|\chi_{i}\right\rangle$ represent the spatial and spin-isospin parts of a nucleon, respectively. The spin-isospin functions $\left|\chi_{i}\right\rangle$ are given by spin-up (or spin-down) protons (or neutrons) in this work. The complex vector $\vec{Z}_{i}$ represents the position of a nucleon in the phase space.

The bare nuclear interactions have the high repulsive potential at a short distance and the non-central potentials to induce the admixture of high orbital angular moments (Wiringa et al., 1995). Every pair wave function in AMD is too simple to describe their induced nucleon-nucleon correlations. Therefore, we construct the effective interactions to be applicable to AMD, G-matrix $(\hat{G})$, starting from bare interactions as

$$
\hat{G}=\hat{v}+\hat{v} \frac{Q}{\varepsilon_{\alpha}+\varepsilon_{\beta}-\left(\hat{t}_{1}+\hat{t}_{2}\right)} \hat{G},
$$

where $\hat{v}, Q, \varepsilon_{i}$ and $\hat{t}_{i}$ represent the bare interaction, the Pauli projection operator, the single-particle energy and kinetic operator, respectively. The above equation called "Bethe-Goldstone equation" denotes an infinite sum of scattering processes of two nucleons in the medium. In this equation, several methods of choosing the energy denominator of the propagator have been proposed. Here, we adopt the "QTQ(gap) choice" (Baldo et al., 2001) where only kinetic energy appears and there is no single-particle potential in intermediate states, as seen in Eq. (2). In the nuclear matter theory, it is known that the convergence of the hole-line expansion in the QTQ choice is inferior to that in the "continuous choice" (Baldo et al., 2001). However, at this stage, we check the adequacy of the QTQ choice in finite nuclei.

In the Brueckner theory, single-particle orbits and energies are needed to determine the G-matrix self-consistently. The single-particle orbits and energies in AMD can be defined by the AMD+Hartree-Fock (AMD-HF) method (Doté et al., 1997). Following AMD-HF, we construct an orthonormal basis $\left\{\tilde{f}_{p}\right\}$ for single-particle orbits by diagonalizing the overlap matrix $B_{i j}$ expressed as

$$
B_{i j}=\left\langle\vec{Z}_{i} \mid \vec{Z}_{j}\right\rangle \cdot\left\langle\chi_{i} \mid \chi_{j}\right\rangle,
$$

and then we have

$$
\begin{gathered}
\sum_{j} B_{i j} \cdot \tilde{C}_{j p}=\mu_{p} \cdot \tilde{C}_{i p}, \quad \sum_{j} \tilde{C}_{j p}^{*} \tilde{C}_{j q}=\delta_{p, q}, \\
\left|\tilde{f}_{p}\right\rangle=\frac{1}{\sqrt{\mu_{p}}} \sum_{j} \tilde{C}_{j p}\left|\vec{Z}_{i}\right\rangle \cdot\left|\chi_{i}\right\rangle, \quad\left\langle\tilde{f}_{p} \mid \tilde{f}_{q}\right\rangle=\delta_{p, q} .
\end{gathered}
$$

The Hartree-Fock Hamiltonian matrix can be written with $\left\{\tilde{f}_{p}\right\}$ :

$$
h_{p q}=\left\langle\tilde{f}_{p}|\hat{t}| \tilde{f}_{q}\right\rangle+\sum_{r}\left\langle\tilde{f}_{p} \tilde{f}_{r}|\hat{G}| \tilde{f}_{q} \tilde{f}_{r}-\tilde{f}_{r} \tilde{f}_{q}\right\rangle .
$$


Then we obtain the single-particle orbit as the solution $\left\{f_{\alpha}\right\}$ for the following equations:

$$
\begin{gathered}
\sum_{q} h_{p q} \cdot g_{q \alpha}=\varepsilon_{\alpha} \cdot g_{p \alpha}, \quad \sum_{q} g_{q \alpha}^{*} g_{q \beta}=\delta_{\alpha, \beta}, \\
\left|f_{\alpha}\right\rangle=\sum_{q} g_{q \alpha}\left|\tilde{f}_{q}\right\rangle=\sum_{j}\left(\sum_{q} \frac{\tilde{C}_{j q}}{\sqrt{\mu_{q}}} \cdot g_{q \alpha}\right)\left|\vec{Z}_{j}\right\rangle \cdot\left|\chi_{j}\right\rangle=\sum_{j} C_{j \alpha}\left|\vec{Z}_{j}\right\rangle \cdot\left|\chi_{j}\right\rangle, \\
\left\langle f_{\alpha} \mid f_{\beta}\right\rangle=\delta_{\alpha, \beta} .
\end{gathered}
$$

And then the single-particle energy is solved as

$$
\varepsilon_{\alpha}=\left\langle f_{\alpha}|\hat{t}| f_{\alpha}\right\rangle+\sum_{\gamma}\left\langle f_{\alpha} f_{\gamma}|\hat{G}| f_{\alpha} f_{\gamma}-f_{\gamma} f_{\alpha}\right\rangle .
$$

In Brueckner-AMD, both the single-particle energies $\left(\varepsilon_{\alpha}\right)$ of the wave function and the G-matrix $(\hat{G})$ are determined self-consistently. That means that the $G$-matrix within the AMD framework can be determined theoretically without any corrections.

\subsection{Details of the G-matrix calculation}

\subsubsection{How to treat the Pauli projection operator}

The Pauli projection operator $Q$ in the Bethe-Goldstone equation, Eq. (2), is introduced in order to take account of the Pauli principle in the scattering processes in the medium. In this framework, the Pauli projection operator $Q$ is represented as

$$
Q=1-P=1-\sum_{\alpha<\beta}\left|f_{\alpha} f_{\beta}\right\rangle\left\langle f_{\alpha} f_{\beta}\right|,
$$

where $P$ is the projection operator for occupied states. In the nuclear matter, the occupied states are represented as the Fermi gas states below the Fermi momentum $k_{F}$ and the operator $Q$ can be defined in terms of $k_{F}$. In Brueckner-AMD, the occupied states are composed of single-particle orbits with AMD single-particle wave functions, and so it is hard to solve the Bethe-Goldstone equation directly because of treating the $Q$-operator. Therefore, following the prescription formulated by Bandō et al. (Bandō et al., 1970), which presents an appropriate means of treating Eq. (2) in the finite nuclear systems, we calculate the G-matrix in the following two steps: First, ignoring the $Q$-operator $(Q \rightarrow 1)$, we solve the equation for $G^{0}$ :

$$
\hat{G}^{0}=\hat{v}+\hat{v} \frac{1}{\varepsilon_{\alpha}+\varepsilon_{\beta}-\left(\hat{t}_{1}+\hat{t}_{2}\right)} \hat{G}^{0} .
$$

Second, we solve the following equation to take into account the $Q$-operator:

$$
\hat{G}=\hat{G}^{0}+\hat{G}^{0} \frac{Q-1}{\varepsilon_{\alpha}+\varepsilon_{\beta}-\left(\hat{t}_{1}+\hat{t}_{2}\right)} \hat{G} .
$$

The explicit form of Eq. (12) becomes the algebraic equations as

$$
\begin{gathered}
\sum_{\alpha<\beta}\left\{\delta_{\gamma_{1}, \alpha} \delta_{\delta_{1}, \beta}+\frac{\left\langle f_{\gamma_{1}} f_{\delta_{1}}\left|\hat{G}^{0}\right| f_{\alpha} f_{\beta}-f_{\beta} f_{\alpha}\right\rangle}{e\left(\gamma_{0} \delta_{0}, \alpha \beta\right)}\right\}\left\langle f_{\alpha} f_{\beta}|\hat{G}| f_{\gamma_{0}} f_{\delta_{0}}-f_{\delta_{0}} f_{\gamma_{0}}\right\rangle \\
=\left\langle f_{\gamma_{1}} f_{\delta_{1}}\left|\hat{G}^{0}\right| f_{\gamma_{0}} f_{\delta_{0}}-f_{\delta_{0}} f_{\gamma_{0}}\right\rangle, \\
e\left(\gamma_{0} \delta_{0}, \alpha \beta\right)=\varepsilon_{\gamma_{0}}+\varepsilon_{\delta_{0}}-\left\langle f_{\alpha}|\hat{t}| f_{\alpha}\right\rangle-\left\langle f_{\beta}|\hat{t}| f_{\beta}\right\rangle .
\end{gathered}
$$




\subsubsection{How to solve the Bethe-Boldstone equation}

In order to see the way how to solve Eq.(11), we use another expression of the Bethe-Goldstone equation with wave functions as

$$
\begin{aligned}
\psi_{k l} & =\varphi_{k l}+\frac{Q}{\varepsilon_{\alpha}+\varepsilon_{\beta}-\left(\hat{t}_{1}+\hat{t}_{2}\right)} \hat{G} \cdot \varphi_{k l} \\
& =\varphi_{k l}+\frac{Q}{\varepsilon_{\alpha}+\varepsilon_{\beta}-\left(\hat{t}_{1}+\hat{t}_{2}\right)} \hat{v} \cdot \psi_{k l},
\end{aligned}
$$

where the following relationship is used:

$$
\left\langle\varphi_{i j}|\hat{G}| \varphi_{k l}\right\rangle=\left\langle\varphi_{i j}|\hat{v}| \psi_{k l}\right\rangle \text {. }
$$

In the above equations, $\psi_{k l}$ and $\varphi_{k l}$ represent the solution of the Bethe-Goldstone equation and the two-body part of the AMD wave function, respectively. As any analogy of Eq. (15), the equation for $G^{0}$, Eq. (11), becomes

$$
\psi_{k l}^{0}=\varphi_{k l}+\frac{1}{\varepsilon_{\alpha}+\varepsilon_{\beta}-\left(\hat{t}_{1}+\hat{t}_{2}\right)} \hat{v} \cdot \psi_{k l}^{0},
$$

where $\psi_{k l}^{0}$ represents the solution of Eq. (11) and it has

$$
\left\langle\varphi_{i j}\left|\hat{G}^{0}\right| \varphi_{k l}\right\rangle=\left\langle\varphi_{i j}|\hat{v}| \psi_{k l}^{0}\right\rangle \text {. }
$$

Then, Eq. (17) can be solved as the differential equation for every pair $(k l)$ of particles:

$$
\left[\hat{T}_{r e l}-\omega\right]\left\{\delta_{l^{\prime}, l_{0}} \cdot \varphi_{l_{0} m}\left(r ; \vec{Z}_{k l}^{r e l}\right)-\psi_{l^{\prime} l_{0} m}^{0 J S}(r)\right\}=\sum_{l^{\prime \prime}} V_{l^{\prime} l^{\prime \prime}}^{J S}(r) \psi_{l^{\prime \prime} l_{0} m}^{0 J S}(r),
$$

where $\hat{T}_{r e l}$ represents the relative kinetic energy operator and $\omega=\varepsilon_{\alpha}+\varepsilon_{\beta}-T_{c m}(i j: k l)$. In this case, $\hat{T}_{r e l}$ can be expressed as

$$
\hat{T}_{r e l}=-\frac{\hbar^{2}}{M} \frac{1}{r} \frac{d^{2}}{d r^{2}} r+\frac{\hbar^{2}}{M} \frac{l^{\prime}\left(l^{\prime}+1\right)}{r^{2}},
$$

and $T_{c m}(i j: k l)$ is the expectation value of the two-body center-of-mass kinetic energy:

$$
T_{c m}(i j: k l)=\frac{\left\langle\vec{Z}_{i j}^{c m}\left|\hat{T}_{c m}\right| \vec{Z}_{k l}^{c m}\right\rangle}{\left\langle\vec{Z}_{i j}^{c m} \mid \vec{Z}_{k l}^{c m}\right\rangle},
$$

where $\hat{T}_{c m}$ represents the two-body center-of-mass kinetic energy operator and $\left|\vec{Z}_{k l}^{c m}\right\rangle$ is the two-body AMD wave function of the center-of-mass part: $\vec{Z}_{k l}^{c m}=\left(\vec{Z}_{k}+\vec{Z}_{l}\right) / \sqrt{2}$. Note that the two-body AMD wave function is decomposed into the center-of-mass and relative parts and its relative one can be applied directly to Eq. (17). In Eq. (19), $\varphi_{l_{0} m}\left(r ; \vec{Z}_{k l}^{r e l}\right)$ represent the two-body AMD wave function of the relative part $\left|\vec{Z}_{k l}^{r e l}\right\rangle: \vec{Z}_{k l}^{r e l}=\left(\vec{Z}_{k}-\vec{Z}_{l}\right) / \sqrt{2}$ for the partial wave having the orbital angular momentum $l_{0}$ and its $z$ component $m$ as

$$
\left\langle\vec{r} \mid \vec{Z}_{k l}^{r e l}\right\rangle=\left(\frac{2 v_{r e l}}{\pi}\right)^{3 / 4} \exp \left[-v_{r e l}\left(\vec{r}-\frac{\vec{Z}_{k l}^{r e l}}{\sqrt{v_{r e l}}}\right)^{2}+\frac{\vec{Z}_{k l}^{r e l 2}}{2}\right]=\sum_{l_{0}, m} \varphi_{l_{0} m}\left(r ; \vec{Z}_{k l}^{r e l}\right)\left|l_{0} m\right\rangle,
$$


where $v_{r e l}=v / 2$ and the explicit expression of $\varphi_{l_{0} m}\left(r ; \vec{Z}_{k l}^{r e l}\right)$ is given later. $\psi_{l^{\prime} l_{0} m}^{0 J S}(r)$ is the solution of Eq. (19) which has the dependence on $(i, j, k, l, \alpha, \beta)$ due to $\omega$ and $\varphi_{l_{0} m}\left(r ; \vec{Z}_{k l}^{r e l}\right)$, and $V_{l l^{\prime}}^{J S}(r)$ is the potential for the two-body channel of total angular momentum $J$ and total spin $S$ with the transition between the angular momenta $l$ and $l^{\prime}$ in the bare interaction $\hat{v}$,

$$
V_{l l^{\prime}}^{J S}(r)=\int_{\text {spin }} \int d \Omega_{\vec{r}} y_{l S J}^{M *}\left(\Omega_{\vec{r}}\right) \hat{v} y_{l^{\prime} S J}^{M}\left(\Omega_{\vec{r}}\right),
$$

where $y_{l S J}^{M}\left(\Omega_{\vec{r}}\right)$ represents the eigenfunction of two-body total angular momentum.

Here, we present explicit expressions of Eq.(19). In the nucleon-nucleon interaction, two-body total angular momentum $J$, total spin $S$, total isospin $T$, and parity are conserved. However, due to the Fermi statistics of two nucleons, two-body states are restricted to the four states under conditions of $S+T+l=o d d$ where $l$ is the two-body angular moment: in the case of even parity $(l=0,2,4, \cdots)$, the spin-triplet $S=1$ state called "triplet-even (3E)" with the isospin-singlet $T=0$ and the spin-singlet $S=0$ state called "singlet-even (1E)" with the isospin-triplet $T=1$; in the case of odd parity $(l=1,3,5, \cdots)$, the spin-triplet $S=1$ state called "triplet-odd (3O)" with the isospin-triplet $T=1$ and the spin-singlet $S=0$ state called "singlet-odd (1O)" with the isospin-singlet $T=0$. Hence, the expressions of Eq. (19) for the above four two-body channels with the lowest allowed angular momentum are presented in the following.

At this stage, one has no unique nucleon-nucleon interaction and many varieties. However, at least, nucleon-nucleon interactions have central $\left(\hat{v}_{c}\right)$, spin-orbit $\left(\hat{v}_{l s}\right)$, and tensor $\left(\hat{v}_{t}\right)$ forces. Here, we consider only the case of the interaction with the above three forces. For spin-singlet states, the interaction has only the central force, and then, Eq. (19) for the singlet-even (1E) with $l=0$ becomes

$$
\left[-\frac{\hbar^{2}}{M} \frac{1}{r} \frac{d^{2}}{d r^{2}} r-\omega\right]\left\{\varphi_{0 m}\left(r ; \vec{Z}_{k l}^{r e l}\right)-\psi_{00 m}^{0 J=0 S=0}(r)\right\}=v_{c}^{(1 \mathrm{E})}(r) \psi_{00 m}^{0 J=0 S=0}(r),
$$

and that for the singlet-odd (1O) with $l=1$ becomes

$$
\left[-\frac{\hbar^{2}}{M} \frac{1}{r} \frac{d^{2}}{d r^{2}} r+\frac{\hbar^{2}}{M} \frac{2}{r^{2}}-\omega\right]\left\{\varphi_{1 m}\left(r ; \vec{Z}_{k l}^{r e l}\right)-\psi_{11 m}^{0 J=1 S=0}(r)\right\}=v_{c}^{(10)}(r) \psi_{11 m}^{0 J=1 S=0}(r) .
$$

For spin-triplet states, nucleon-nucleon interactions include central, spin-orbit, and tensor forces. Note that the orbital angular momentum $l$ state may be admixed with higher angular momentum $l^{\prime}$ state by the tensor force. Hence, Eq. (19) for the triplet-even (3E) with $l=0$ becomes the coupled equation to $l^{\prime}=2$ as

$$
\begin{aligned}
& {\left[-\frac{\hbar^{2}}{M} \frac{1}{r} \frac{d^{2}}{d r^{2}} r-\omega\right]\left\{\varphi_{0 m}\left(r ; \vec{Z}_{k l}^{r e l}\right)-\psi_{00 m}^{0 J=1 S=1}(r)\right\}=2 \sqrt{2} v_{t}^{(3 \mathrm{E})}(r) \psi_{20 m}^{0 J=1 S=1}(r) } \\
& +v_{c}^{(3 \mathrm{E})}(r) \psi_{00 m}^{0 J=1 S=1}(r), \\
- & {\left[-\frac{\hbar^{2}}{M} \frac{1}{r} \frac{d^{2}}{d r^{2}} r+\frac{\hbar^{2}}{M} \frac{6}{r^{2}}-\omega\right] \psi_{20 m}^{0 J=1 S=1}(r)=2 \sqrt{2} v_{t}^{(3 \mathrm{E})}(r) \psi_{00 m}^{0 J=1 S=1}(r) } \\
& +\left[v_{c}^{(3 \mathrm{E})}(r)-3 v_{l s}^{(3 \mathrm{E})}(r)-2 v_{t}^{(3 \mathrm{E})}(r)\right] \psi_{20 m}^{0 J=1 S=1}(r) .
\end{aligned}
$$


For the the triplet-odd (3O) with $l=1$, the cases of $J=0,1$, and 2 are considered. And then, Eq. (19) for the 30 with $J=0$ and $l=1$ becomes

$$
\begin{aligned}
& {\left[-\frac{\hbar^{2}}{M} \frac{1}{r} \frac{d^{2}}{d r^{2}} r+\frac{\hbar^{2}}{M} \frac{2}{r^{2}}-\omega\right]\left\{\varphi_{1 m}\left(r ; \vec{Z}_{k l}^{r e l}\right)-\psi_{11 m}^{0 J=0 S=1}(r)\right\}} \\
& =\left[v_{c}^{(30)}(r)-2 v_{l s}^{(30)}(r)-4 v_{t}^{(30)}(r)\right] \psi_{11 m}^{0 J=0 S=1}(r),
\end{aligned}
$$

that for the $3 \mathrm{O}$ with $J=1$ and $l=1$ becomes

$$
\begin{aligned}
& {\left[-\frac{\hbar^{2}}{M} \frac{1}{r} \frac{d^{2}}{d r^{2}} r+\frac{\hbar^{2}}{M} \frac{2}{r^{2}}-\omega\right]\left\{\varphi_{1 m}\left(r ; \vec{Z}_{k l}^{r e l}\right)-\psi_{11 m}^{0 J=1 S=1}(r)\right\}} \\
& =\left[v_{c}^{(30)}(r)-v_{l s}^{(30)}(r)+2 v_{t}^{(30)}(r)\right] \psi_{11 m}^{0 J=1 S=1}(r),
\end{aligned}
$$

and that for the $3 \mathrm{O}$ with $J=2$ and $l=1$ becomes the coupled equation to $l^{\prime}=3$ as

$$
\begin{aligned}
& {\left[-\frac{\hbar^{2}}{M} \frac{1}{r} \frac{d^{2}}{d r^{2}} r+\frac{\hbar^{2}}{M} \frac{2}{r^{2}}-\omega\right]\left\{\varphi_{1 m}\left(r ; \vec{Z}_{k l}^{r e l}\right)-\psi_{11 m}^{0 J=2 S=1}(r)\right\}=\frac{6 \sqrt{6}}{5} v_{t}^{(30)}(r) \psi_{31 m}^{0 J=2 S=1}(r)} \\
& +\left[v_{c}^{(30)}(r)+v_{l s}^{(30)}(r)-\frac{2}{5} v_{t}^{(30)}(r)\right] \psi_{11 m}^{0 J=2 S=1}(r), \\
& -\left[-\frac{\hbar^{2}}{M} \frac{1}{r} \frac{d^{2}}{d r^{2}} r+\frac{\hbar^{2}}{M} \frac{12}{r^{2}}-\omega\right] \psi_{31 m}^{0 J=2 S=1}(r)=\frac{6 \sqrt{6}}{5} v_{t}^{(30)}(r) \psi_{11 m}^{0 J=2 S=1}(r) \\
& +\left[v_{c}^{(30)}(r)-4 v_{l s}^{(30)}(r)-\frac{8}{5} v_{t}^{(30)}(r)\right] \psi_{31 m}^{0 J=2 S=1}(r) .
\end{aligned}
$$

In the following, we explain the partial-wave expansion of the two-body AMD wave function of the relative part in Eq. (22). It becomes

$$
\left\langle\vec{r} \mid \vec{Z}_{k l}^{r e l}\right\rangle=\left(\frac{2 v_{r e l}}{\pi}\right)^{3 / 4} \exp \left[-v_{r e l} \vec{r}^{2}-\frac{\vec{Z}_{k l}^{r e l} 2}{2}\right] \cdot \exp \left[2 \sqrt{v_{r e l}} \vec{Z}_{k l}^{r e l} \cdot \vec{r}\right],
$$

where the complex vector $\vec{Z}_{k l}^{r e l}=\vec{Z}_{R}+i \vec{Z}_{I}$. Here, the term "exp $\left[2 \sqrt{v_{r e l}} \vec{Z}_{k l}^{r e l} \cdot \vec{r}\right]$ " can be expanded as multipole:

$$
\begin{aligned}
\exp \left[2 \sqrt{v_{r e l}} \vec{Z}_{k l}^{r e l} \cdot \vec{r}\right]= & (4 \pi)^{2} \sum_{l_{1}, l_{2}, m_{1}, m_{2}} J_{l_{1}}\left(2 \sqrt{v_{r e l}} Z_{R} r\right) \cdot i^{l_{2}} j_{l_{2}}\left(2 \sqrt{v_{r e l}} Z_{I} r\right) \times \\
& Y_{l_{1} m_{1}}^{*}\left(\hat{\mathbf{Z}}_{R}\right) Y_{l_{2} m_{2}}^{*}\left(\hat{\mathbf{Z}}_{I}\right) Y_{l_{1} m_{1}}(\hat{\boldsymbol{r}}) Y_{l_{2} m_{2}}(\hat{\boldsymbol{r}}),
\end{aligned}
$$

where $Z_{R, I}=\left|\vec{Z}_{R, I}\right|$ and $\hat{Z}_{R, I}=\vec{Z}_{R, I} /\left|\vec{Z}_{R, I}\right|$. In Eq. (31), we use the formulas

$$
\begin{aligned}
& \exp [\overrightarrow{i k} \cdot \vec{r}]=4 \pi \sum_{l, m} i^{l} j_{l}(k r) Y_{l m}^{*}(\hat{\boldsymbol{k}}) Y_{l m}(\hat{\boldsymbol{r}}), \\
& \exp [\vec{k} \cdot \overrightarrow{\boldsymbol{r}}]=4 \pi \sum_{l, m} J_{l}(k r) Y_{l m}^{*}(\hat{\boldsymbol{k}}) Y_{l m}(\hat{\boldsymbol{r}}),
\end{aligned}
$$

where $J_{l}(r) \equiv(-i)^{l} j_{l}(i r)$. We use the following formula for the product of two spherical harmonics with $3-j$ symbols:

$$
Y_{l_{1} m_{1}}(\hat{\boldsymbol{r}}) Y_{l_{2} m_{2}}(\hat{\boldsymbol{r}})=\sum_{l, m} \sqrt{\frac{\left(2 l_{1}+1\right)\left(2 l_{2}+1\right)(2 l+1)}{4 \pi}}\left(\begin{array}{ccc}
l_{1} & l_{2} & l \\
m_{1} & m_{2} & m
\end{array}\right)\left(\begin{array}{ccc}
l_{1} & l_{2} & l \\
0 & 0 & 0
\end{array}\right) Y_{l m}^{*}(\hat{\boldsymbol{r}}) .
$$




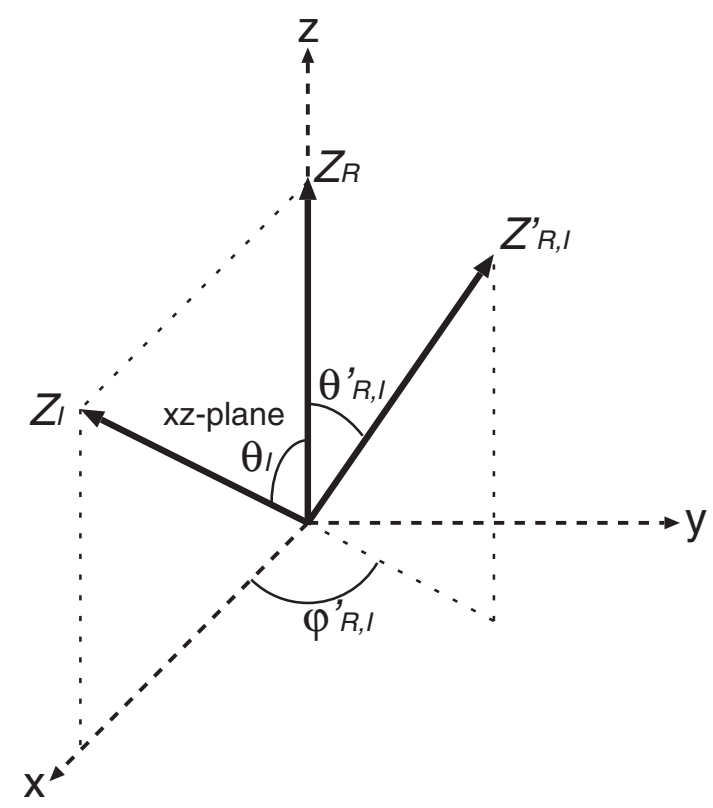

Fig. 1. The vectors $\vec{Z}_{k l}^{r e l}=\vec{Z}_{R}+i \vec{Z}_{I}$ and $\vec{Z}_{i j}^{r e l}=\vec{Z}_{R}^{\prime}+i \vec{Z}_{I}^{\prime}$ in the body-fixed frame we adopt in this framework are schematically shown.

Thus, in the calculations of $\left\langle\vec{Z}_{i j}^{r e l}|\hat{O}| \vec{Z}_{k l}^{r e l}\right\rangle$, the state of $\left|\vec{Z}_{k l}^{r e l}\right\rangle$ can be expressed as the expansion of angular momental states $|\operatorname{lm}\rangle$ :

$$
\begin{aligned}
\left\langle\vec{r} \mid \vec{Z}_{k l}^{r e l}\right\rangle & =\sum_{l, m} \varphi_{l m}\left(r ; \vec{Z}_{k l}^{r e l}\right)|l m\rangle \\
& =\left(\frac{2 v_{r e l}}{\pi}\right)^{3 / 4} \exp \left[-v_{r e l} \vec{r}^{2}-\frac{\vec{Z}_{k l}^{r e l} 2}{2}\right] \sum_{l, m} z_{l m}\left(2 \sqrt{v_{r e l}} r ; \vec{Z}_{k l}^{r e l}\right)|l m\rangle,
\end{aligned}
$$

where

$$
\begin{aligned}
z_{l m}\left(r ; \vec{Z}_{k l}^{r e l}\right) \equiv & (4 \pi)^{3 / 2} \sum_{l_{1}, l_{2}, m_{1}, m_{2}} \sqrt{\left(2 l_{1}+1\right)\left(2 l_{2}+1\right)} J_{l_{1}}\left(Z_{R} r\right) \cdot i^{l_{2}} j_{l_{2}}\left(Z_{I} r\right) \times \\
& Y_{l_{1} m_{1}}^{*}\left(\hat{\mathbf{Z}}_{R}\right) Y_{l_{2} m_{2}}^{*}\left(\hat{\mathbf{Z}}_{I}\right) \sqrt{2 l+1}\left(\begin{array}{ccc}
l_{1} & l_{2} & l \\
m_{1} & m_{2} & m
\end{array}\right)\left(\begin{array}{ccc}
l_{1} & l_{2} & l \\
0 & 0 & 0
\end{array}\right) .
\end{aligned}
$$

In the case of the rotational-invariant operator, for example, nucleon-nucleon interaction, its expectation value may be calculated in the arbitrary body-fixed frame because $\left\langle\vec{Z}_{i j}^{r e l}|\hat{O}| \vec{Z}_{k l}^{r e l}\right\rangle=\left\langle\vec{Z}_{i j}^{r e l}\left|\hat{R}^{\dagger}(\Omega) \hat{O} \hat{R}(\Omega)\right| \vec{Z}_{k l}^{r e l}\right\rangle$ where $\hat{R}(\Omega)$ is the operator of rotation by the arbitrary Euler angle $\Omega$. Here, we adopt the body-fixed frame shown in Fig. 1 where $\vec{Z}_{R}$ is fixed along the $z$-axis with the angle $\hat{Z}_{R}=\left(\theta_{R}=0, \varphi_{R}=0\right)$ and $\vec{Z}_{I}$ is in the $x z$-plane with the angle $\hat{\mathbf{Z}}_{I}=\left(\theta_{I}, \varphi_{I}=0\right)$. 


\subsubsection{G-matrix calculations with correlation functions}

In order to explain the way how to calculate the G-matrix element, we provide another viewpoint of Eq. (15):

$$
\psi_{k l}=\varphi_{k l}+\frac{Q}{\varepsilon_{\alpha}+\varepsilon_{\beta}-\left(\hat{t}_{1}+\hat{t}_{2}\right)} \hat{G} \cdot \varphi_{k l} \equiv \hat{F}_{k l} \cdot \varphi_{k l},
$$

where we call $\hat{F}_{k l}$ the "correlation function" defined schematically as $\hat{F}_{k l}=\psi_{k l} / \varphi_{k l}$ which represents nucleon-nucleon correlations induced by the interaction. From Eqs. (16) and (36), one can obtain the G-matrix element as

$$
\left\langle\varphi_{i j}|\hat{G}| \varphi_{k l}\right\rangle=\left\langle\varphi_{i j}\left|\hat{v} \cdot \hat{F}_{k l}\right| \varphi_{k l}\right\rangle .
$$

The equation for $\hat{G}^{0}$ has the analogy to Eq. (37):

$$
\left\langle\varphi_{i j}\left|\hat{G}^{0}\right| \varphi_{k l}\right\rangle=\left\langle\varphi_{i j}\left|\hat{v} \cdot \hat{F}_{k l}^{0}\right| \varphi_{k l}\right\rangle,
$$

where the correlation function $\hat{F}_{k l}^{0}$ is defined as $\hat{F}_{k l}^{0}=\psi_{k l}^{0} / \varphi_{k l}$ by using the solution $\psi_{k l}^{0}$ of Eq. (17). Hence, $\hat{G}^{0}$ for each $J$ and $S$ channel becomes

$$
G_{l_{0} l^{\prime}}^{J S}(r)=\sum_{l^{\prime \prime}} V_{l^{\prime} l^{\prime \prime}}^{J S}(r) F_{k l}^{0}\left[\begin{array}{l}
J S \\
l^{\prime \prime} l_{0}
\end{array}\right](\omega, r)
$$

where the correlation function is given by $\psi_{l^{\prime} l_{0} m}^{0 J S}(r)$ and $\varphi_{l_{0} m}\left(r ; \vec{Z}_{k l}^{r e l}\right)$ in Eq. (19) as

$$
F_{k l}^{0}\left[\begin{array}{c}
J S \\
l^{\prime} l_{0}
\end{array}\right](\omega, r)=\psi_{l^{\prime} l_{0} m}^{0 J S}(r) / \varphi_{l_{0} m}\left(r ; \vec{Z}_{k l}^{r e l}\right) .
$$

Note that the above correlation function has the dependence on $(i, j, k, l, \alpha, \beta)$ through $\varphi_{l_{0} m}\left(r ; \vec{Z}_{k l}^{r l}\right)$ and $\omega=\varepsilon_{\alpha}+\varepsilon_{\beta}-T_{c m}(i j: k l)$ of Eq. (19). The correlation function of Eq. (40) is approximated as $m=0$ in $\psi_{l^{\prime} l_{0} m}^{0 J S}(r)$ and $\varphi_{l_{0} m}\left(r ; \vec{Z}_{k l}^{r e l}\right)$ because we find that it has little dependence on $m$. In order to carry the practical calculations, we determine $\hat{G}^{0}$ with explicit operators as follows:

$$
\hat{G}_{l_{0} l^{\prime}}^{S}=\sum_{\lambda=0}^{2} G^{0 S}{ }_{l_{0} l^{\prime}, \lambda}(r)\left(\boldsymbol{R}_{\lambda} \cdot \boldsymbol{S}_{\lambda}\right),
$$

where $\left(\boldsymbol{R}_{\lambda} \cdot S_{\lambda}\right)$ is a scalar product of an orbital tensor $\boldsymbol{R}_{\lambda}$ and a spin tensor $S_{\lambda}$ of rank $\lambda$. The components of $\lambda=0,1$, and 2 correspond to the central, spin-orbit, and tensor forces in a potential, respectively. And then, one can utilize the following equation

$$
G^{0}{ }_{l_{0} l^{\prime}, \lambda}^{S}(r)=\frac{(2 \lambda+1)}{\left\langle l_{0}\left\|\boldsymbol{R}_{\lambda}\right\| l^{\prime}\right\rangle\left\langle S\left\|S_{\lambda}\right\| S\right\rangle} \sum_{J}(-1)^{J-l_{0}-S}(2 J+1) W\left(l_{0} l^{\prime} S S ; \lambda J\right) G_{l_{0} l^{\prime}}^{0 J}(r),
$$

where the expressions with double bars such as $\left\langle l_{0}\left\|\boldsymbol{R}_{\lambda}\right\| l^{\prime}\right\rangle$ represent the reduced matrix elements and $W\left(l_{0} l^{\prime} S S ; \lambda J\right)$ is the Racah coefficient. In this calculation, the angular momenta $l_{0}$ and $l^{\prime}$ in Eq. (42) are taken as the allowed lowest values for each two-body channel: $l_{0}=l^{\prime}=0$ in the $1 \mathrm{E}$ and $3 \mathrm{E}$ central force, $l_{0}=l^{\prime}=1$ in all forces of the $1 \mathrm{O}$ and $3 \mathrm{O}$ channels, $l_{0}=l^{\prime}=2$ in the $3 \mathrm{E}$ spin-orbit force, and $l_{0}=0, l^{\prime}=2$ in the $3 \mathrm{E}$ tensor force. As a result, $\hat{G}^{0}$ becomes

$$
\hat{G}^{0}=G_{\lambda=0}^{0}(r)+G_{\lambda=1}^{0}(r) \vec{L} \cdot \vec{S}+G_{\lambda=2}^{0}(r) S_{12} .
$$


Note that $\hat{G}^{0}$ has the dependence on $(i, j, k, l, \alpha, \beta)$ drived from the correlation function of Eq. (40). By using the matrix element for $\hat{G}^{0},\left\langle f_{\gamma} f_{\delta}\left|\hat{G}^{0}\right| f_{\alpha} f_{\beta}-f_{\beta} f_{\alpha}\right\rangle$, one can obtain the G-matrix element $\left\langle f_{\gamma} f_{\delta}|\hat{G}| f_{\alpha} f_{\beta}-f_{\beta} f_{\alpha}\right\rangle$ from Eq. (13).

The $G$-matrix has the dependence on the bra state $(i, j)$ and difficulty to treat the case of superpostion of wave functions. In addition, the $G$-matrix is given by the matrix element not to be easy to analyze its properties. Hence, in the following, we present the practical alternative. In Eq. (19), we use $T_{c m}(k l)$ instead of $T_{c m}(i j: k l)$ in $\omega$ as

$$
T_{c m}(k l)=\frac{\left\langle\vec{Z}_{k l}^{c m}\left|\hat{T}_{c m}\right| \vec{Z}_{k l}^{c m}\right\rangle}{\left\langle\vec{Z}_{k l}^{c m} \mid \vec{Z}_{k l}^{c m}\right\rangle},
$$

which reprents the expectation value of the two-body center-of-mass kinetic energy for only the ket state. We solve Eq. (19) using $\omega_{0}=\varepsilon_{\alpha}+\varepsilon_{\beta}-T_{c m}(k l)$ instead of $\omega$, and then, by using its solution, we determine the correlation function $F_{k l}^{0}\left[\begin{array}{l}J S \\ l^{\prime} l_{0}\end{array}\right]\left(\omega_{0}, r\right)$ with no dependence on the bra state $(i, j)$. One can obtain the alternative of the $G^{0}$-matrix, $\left\langle f_{\gamma} f_{\delta}\left|\hat{G}^{\prime 0}\right| f_{\alpha} f_{\beta}-f_{\beta} f_{\alpha}\right\rangle$, by applying Eqs. (39) and (42) with the correlation function $F_{k l}^{0}\left[\begin{array}{l}J S \\ l^{\prime} l_{0}\end{array}\right]\left(\omega_{0}, r\right)$. Here, we consider the ratio of the G-matrix element solved by $\left\langle f_{\alpha} f_{\beta}\left|\hat{G}^{0}\right| f_{\alpha} f_{\beta}-f_{\beta} f_{\alpha}\right\rangle$ with $F_{k l}^{0}\left[\begin{array}{l}J_{l^{\prime} l_{0}} \\ ]\end{array}\right](\omega, r)$ from Eq. (13), $\left\langle f_{\alpha} f_{\beta}|\hat{G}| f_{\alpha} f_{\beta}-f_{\beta} f_{\alpha}\right\rangle$, to the matrix element $\left\langle f_{\alpha} f_{\beta}\left|\hat{G}^{\prime 0}\right| f_{\alpha} f_{\beta}-f_{\beta} f_{\alpha}\right\rangle$ :

$$
C_{Q}^{\alpha \beta}=\frac{\left\langle f_{\alpha} f_{\beta}|\hat{G}| f_{\alpha} f_{\beta}-f_{\beta} f_{\alpha}\right\rangle}{\left\langle f_{\alpha} f_{\beta}\left|\hat{G}^{\prime 0}\right| f_{\alpha} f_{\beta}-f_{\beta} f_{\alpha}\right\rangle},
$$

which represents the effects of the operator $Q$ and $T_{c m}(i j: k l)$. By using the above value $C_{Q}^{\alpha \beta}$, one can present the explicit form of the correlation function $\hat{F}_{k l}$ in Eq. (36) as

$$
F_{k l}\left[\begin{array}{l}
J S \\
l^{\prime} l_{0}
\end{array}\right]\left(\omega_{0}, r\right)=C_{Q}^{\alpha \beta} \cdot F_{k l}^{0}\left[\begin{array}{l}
J S \\
l^{\prime} l_{0}
\end{array}\right]\left(\omega_{0}, r\right) .
$$

Hence, the G-matrix for each $J$ and $S$ channel becomes

$$
G_{l_{0} l^{\prime}}^{J S}(r)=\sum_{l^{\prime \prime}} V_{l^{\prime} l^{\prime \prime}}^{J S}(r) F_{k l}\left[\begin{array}{l}
J S \\
l^{\prime \prime} l_{0}
\end{array}\right]\left(\omega_{0}, r\right)
$$

Applying Eq. (42) with $G_{l_{0} l^{\prime}}^{J S}(r)$ instead of $G^{0}{ }_{l_{0} l^{\prime}}^{J S}(r)$, the G-matrix can be determined as

$$
\hat{G}=G_{\lambda=0}(r)+G_{\lambda=1}(r) \vec{L} \cdot \vec{S}+G_{\lambda=2}(r) S_{12} .
$$

Note that the expectation value for the potential of Eq. (48) reproduces the G-matrix element. As seen in the above discussion, one can obtain the $G$-matrix as the effective interaction with the explicit form by using the correlation function of Eq. (46).

\subsubsection{Explicit examples of G-matrix}

In order to see the properties of G-matrix in Brueckner-AMD, we present the G-matrix in the specific case. As the bare nucleon-nucleon interaction, we adopt the Argonne $v 8^{\prime}\left(\mathrm{AV} 8^{\prime}\right)$ (Wiringa \& Pieper, 2002). AV8 ${ }^{\prime}$ is constructed by renormalizing the $\vec{L}^{2}$ and $(\vec{L} \cdot \vec{S})^{2}$ terms in the Argonne v18 (AV18) (Wiringa et al., 1995), which is the high accurate bare nucleon-nucleon 
interaction, into the central, spin-orbit, and tensor forces. And the iso-spin symmetry and independence breaking included in AV18 are ignored in the case of AV8' . In the following, we switch off the electromagnetic interactions.

As a preparation, several explicit terms of the G-matrix in Eq. (48) are shown. For the spin-singlet states, the G-matrix has only the central force given by the simple product of the bare interaction multiplied by the correlation function. For example, the singlet-even (1E) central force of the G-matrix becomes

$$
G_{c}^{(1 \mathrm{E})}(r)=v_{c}^{(1 \mathrm{E})}(r) \cdot F_{k l}\left[\begin{array}{l}
J=0 S=0 \\
l^{\prime}=0 l_{0}=0
\end{array}\right]\left(\omega_{0}, r\right) .
$$

The singlet-odd (1O) central G-matrix is given as the same way as the above equation. On the other hand, for the spin-triplet states, the non-central forces in the bare interaction are renormalized into the central G-matrix through the correlation functions. Hence, the triplet-even (3E) central G-matrix becomes

$$
G_{c}^{(3 \mathrm{E})}(r)=v_{c}^{(3 \mathrm{E})} \cdot F_{k l}\left[\begin{array}{l}
J=1 S=1 \\
l^{\prime}=0 l_{0}=0
\end{array}\right]+2 \sqrt{2} v_{t}^{(3 \mathrm{E})} \cdot F_{k l}\left[\begin{array}{l}
J=1 S=1 \\
l^{\prime}=2 l_{0}=0
\end{array}\right],
$$

and the triplet-odd (3O) central becomes

$$
\begin{aligned}
G_{c}^{(30)}(r)=\frac{1}{9} & \left\{\left(v_{c}^{(30)}-2 v_{l s}^{(30)}-4 v_{t}^{(30)}\right) \cdot F_{k l}\left[\begin{array}{l}
J=0 S=1 \\
l^{\prime}=1 l_{0}=1
\end{array}\right]\right. \\
& +3\left(v_{c}^{(30)}-v_{l s}^{(30)}+2 v_{t}^{(30)}\right) \cdot F_{k l}\left[\begin{array}{l}
J=1 S=1 \\
l^{\prime}=1 l_{0}=1
\end{array}\right] \\
& \left.+5\left(v_{c}^{(30)}+v_{l s}^{(30)}-\frac{2}{5} v_{t}^{(30)}\right) \cdot F_{k l}\left[\begin{array}{l}
J=2 S=1 \\
l^{\prime}=1 l_{0}=1
\end{array}\right]+6 \sqrt{6} v_{t}^{(30)} \cdot F_{k l}\left[\begin{array}{l}
J=2 S=1 \\
l^{\prime}=3 l_{0}=1
\end{array}\right]\right\},
\end{aligned}
$$

where the $\left(\omega_{0}, r\right)$ dependence in the above right hands is omitted for simplicity. In addition, as examples, for the $3 \mathrm{E}$ tensor and $3 \mathrm{O}$ spin-orbit G-matrices, their expressions where the $\left(\omega_{0}, r\right)$ dependence is omitted as the aboves are given:

$$
\begin{aligned}
G_{t}^{(3 \mathrm{E})}(r)= & v_{t}^{(3 \mathrm{E})} \cdot F_{k l}\left[\begin{array}{l}
J=1 S=1 \\
l^{\prime}=0 l_{0}=0
\end{array}\right]+\frac{1}{2 \sqrt{2}}\left(v_{c}^{(3 \mathrm{E})}-3 v_{l s}^{(3 \mathrm{E})}-2 v_{t}^{(3 \mathrm{E})}\right) \cdot F_{k l}\left[\begin{array}{l}
J=1 S=1 \\
l^{\prime}=2 l_{0}=0
\end{array}\right], \\
G_{l s}^{(3 \mathrm{O})}(r)=-\frac{1}{12} & \left\{2\left(v_{c}^{(3 \mathrm{O})}-2 v_{l s}^{(3 \mathrm{O})}-4 v_{t}^{(3 \mathrm{O})}\right) \cdot F_{k l}\left[\begin{array}{l}
J=0 S=1 \\
l^{\prime}=1 l_{0}=1
\end{array}\right]\right. \\
& +3\left(v_{c}^{(3 \mathrm{O})}-v_{l s}^{(3 \mathrm{O})}+2 v_{t}^{(3 \mathrm{O})}\right) \cdot F_{k l}\left[\begin{array}{l}
J=1 S=1 \\
l^{\prime}=1 l_{0}=1
\end{array}\right] \\
& \left.-5\left(v_{c}^{(3 \mathrm{O})}+v_{l s}^{(3 \mathrm{O})}-\frac{2}{5} v_{t}^{(3 \mathrm{O})}\right) \cdot F_{k l}\left[\begin{array}{l}
J=2 S=1 \\
l^{\prime}=1 l_{0}=1
\end{array}\right]-6 \sqrt{6} v_{t}^{(3 \mathrm{O})} \cdot F_{k l}\left[\begin{array}{l}
J=2 S=1 \\
l^{\prime}=3 l_{0}=1
\end{array}\right]\right\} .
\end{aligned}
$$

In Brueckner-AMD, the G-matrix for each pair have dependence on not only the paricle pair $(k, l)$ but also the single-particle orbits $(\alpha, \beta)$ due to the dependence on $\omega_{0}=\varepsilon_{\alpha}+\varepsilon_{\alpha}-T_{c m}(k l)$ in the correlation function. Therefore, in general, one cannot abstract the explicit form as the interaction between particles $(k, l)$. However, as the special case, in the system of ${ }^{4} \mathrm{He}$ consisting of the spin-up proton, spin-up neutron, spin-down proton, and spin-down neutron, the G-matrix is given as the interaction between particles $(k, l)$ because the single-particle orbits $\left|f_{\alpha, \beta}\right\rangle$ are equal to the one-particle wave funcions, which means $(\alpha, \beta)=(k, l)$, on condition that the overlap matrix $B_{i j}$ in Eq. (3) becomes a diagonal matrix such as this system. Here, we consider the G-matrix between particles in the $3 \mathrm{~N}-\mathrm{N}$ cluster system of ${ }^{4} \mathrm{He}$ where 

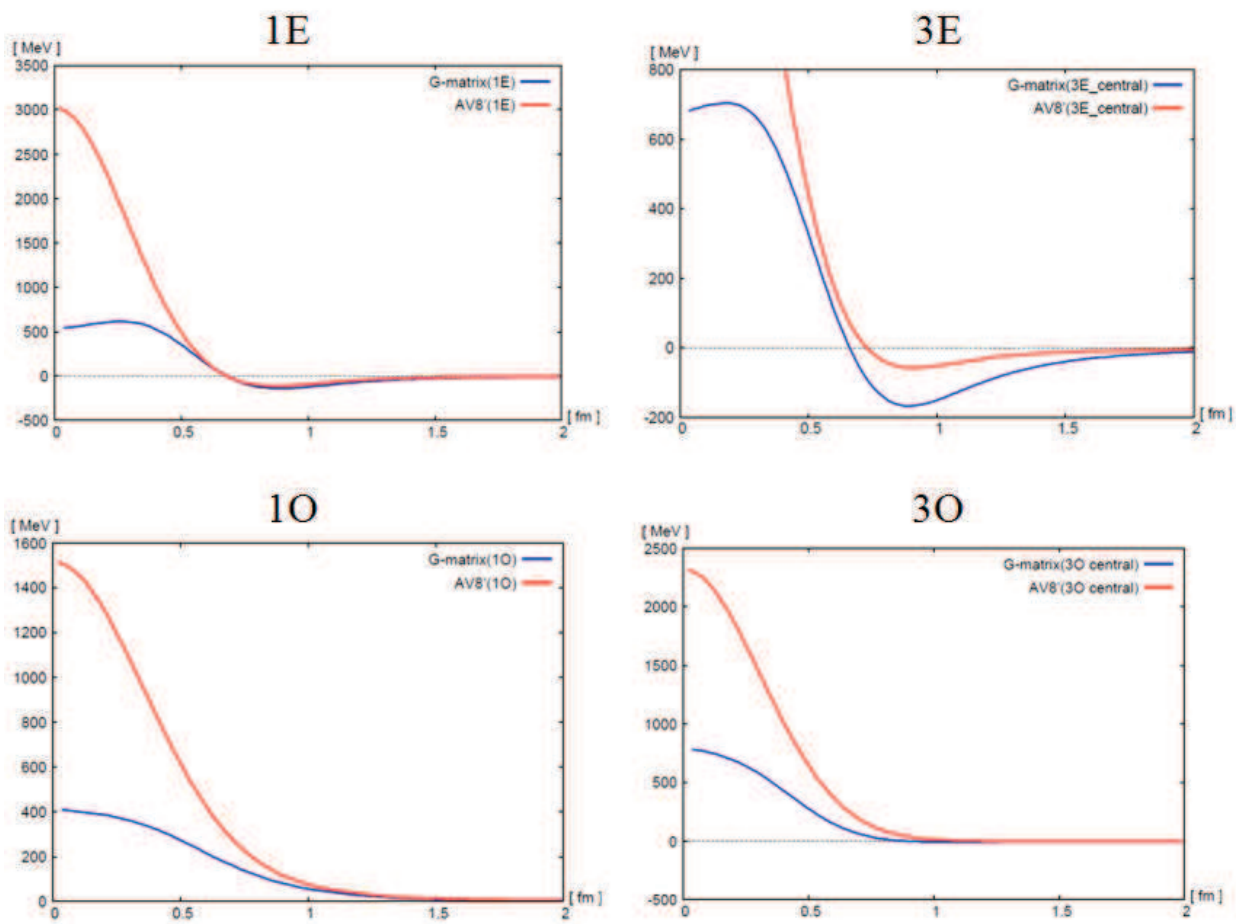

Fig. 2. The comparison of the G-matrix with the bare interaction AV8' for the central components of two-body four channels. In these graphs, the $x$ - and $y$-axes represent the distances between nucleons ( $\mathrm{fm}$ ) and the potential energies $(\mathrm{MeV})$, respectively. The blue and red lines represent the $\mathrm{G}$-matrix and $\mathrm{AV} 8^{\prime}$, respectively. The explanation of the G-matrix is in text in detail. In the $3 \mathrm{E}$ channel, the attractive part is enlarged.

three nucleons $(3 \mathrm{~N})$ located at the same positon are separated from one nucleon $(\mathrm{N})$. We assume that three nucleons consist of the spin-up proton, spin-up neutron, and spin-down proton and one nucleon to be sperated is the spin-down neutron, and the distance between three nucleons and one nucleon is $1.0 \mathrm{fm}$. The AMD wave funciton of Eq. (1) with $v=0.238$ $\left(\mathrm{fm}^{-2}\right)$ is used in this calculation. In Figs. 2 and 3, we present all forces of the G-matrix between the spin-up proton in three nucleons and the spin-down neutron to be one nucleon sperated from three nucleons at a distance of $1.0 \mathrm{fm}$ and compare them with those of the bare interaction $\mathrm{AV}^{\prime}$. As seen in Fig. 2, the central forces of the bare interaction AV8' have huge repulsive parts at a short distance, while, in the central G-matrices, the short range repulsion is reduced to be applicable to the AMD wave function. At a long distance, each force of the $\mathrm{G}$-matrix except the $3 \mathrm{E}$ state agrees with the behaviour of the bare interaction. For the 3E $G$-matrices, the renormalization of the strong $3 \mathrm{E}$ tensor force with a long range tail in the bare interaction acts as the long range correlation to be large contributions. Especially, in the 3E central G-matrix, the renormalization of tensor force by the correlation for the admixture of $l^{\prime}=2, F_{k l}\left[\begin{array}{l}J=1 S=1 \\ l^{\prime}=2 l_{0}=0\end{array}\right]$ in Eq. (50), induces the more attractive part than that of the bare interaction and contributes largely to the binding energy of a nucleus. 

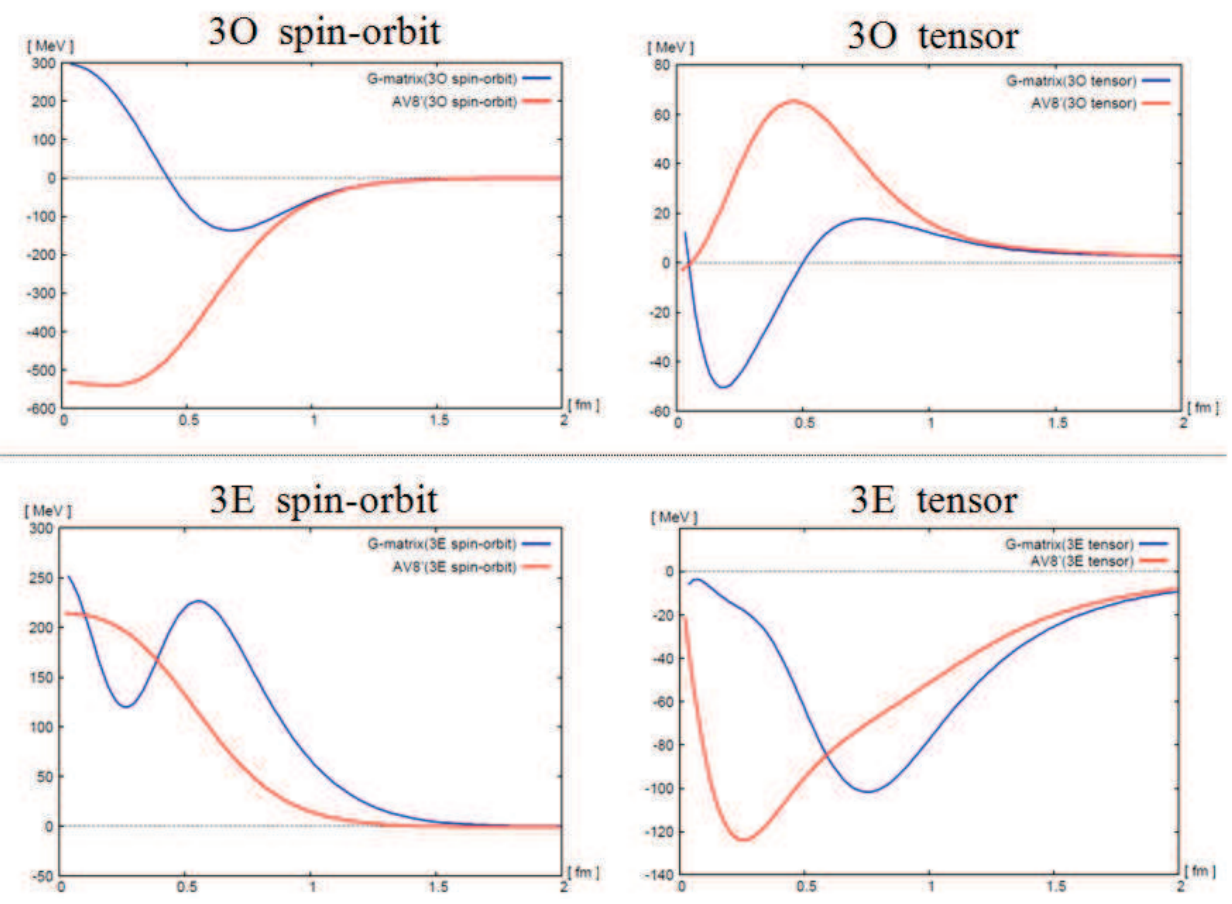

Fig. 3. The comparison of the G-matrix with the bare interaction AV8' for the non-central components of $3 \mathrm{O}$ and $3 \mathrm{E}$ channels in the upper and lower panels, respectively. In these graphs, the meanings of the axes and lines are the same as Fig. 2.

\subsection{Energy variation in Brueckner-AMD}

In the AMD method, the wave-packet positions $\vec{Z}_{i}$ in Eq. (1) are complex variational parameters in the $A$-nucleons wave function. In this method, one usually sets the initial configuration of the wave-packet positions $\left\{\vec{Z}_{i}\right\}$ randomly and obtains the optimized solution automatically with no assumption by using the frictional cooling method of molecular dynamics (Kanada-En'yo et al., 1995) to determine $\vec{Z}_{i}$. The brief expression of frictional cooling equation is given by

$$
\frac{d \vec{Z}_{i}}{d t}=-\frac{\partial\langle H\rangle}{\partial \vec{Z}_{i}^{*}}, \quad \frac{d \vec{Z}_{i}^{*}}{d t}=-\frac{\partial\langle H\rangle}{\partial \vec{Z}_{i}},
$$

where $\langle H\rangle$ is the expectation value of Hamiltonian. In Brueckner-AMD, the expectation value of Hamiltonian is expressed as $\langle H\rangle=\langle T\rangle-\left\langle T_{C M}\right\rangle+\langle G\rangle$ where $\langle T\rangle,\left\langle T_{C M}\right\rangle$, and $\langle G\rangle$ represent the expectation values of the $A$-body summation of the one-body kinetic energy operator $\hat{t}_{i}$ as $\hat{T}=\sum_{i}^{A} \hat{t}_{i}$, the center-of-mass kinetic energy of the system, and the G-matrix as two-body interactions, respectively. In Eq. (54), the next positions of $\vec{Z}_{i}$ in the time evolution are decided as

$$
\vec{Z}_{i}(t+\Delta t)=\vec{Z}_{i}(t)-\Delta t \cdot \frac{\partial\langle H\rangle}{\partial \vec{Z}_{i}^{*}} .
$$


If $\langle H\rangle$ converges in this time evolution, we consider that the optimized energy and state are attained. In Brueckner-AMD, the derivative of $\vec{Z}_{i}$ in $\langle H\rangle$ cannot be calculated analytically because the G-matrix depends on the change of the single-particle orbits and the Pauli projection operator derived from the configuration of the wave-packet positions $\left\{\vec{Z}_{i}\right\}$. Therefore, we determine the $G$-matrix self-consistently for each small displaced configuration of $\left\{\vec{Z}_{i}+\Delta \vec{Z}_{i}\right\}$ and calculate this derivative as the difference in $\vec{Z}_{i}$.

\section{Applications}

We apply Brueckner-AMD to various light nuclei and present the applicability of this method. In the subsection 3.1, we present the results of light nuclei with the mass number $A<10$ to describe cluster structures and reproduce the energy level schemes in Brueckner-AMD. Furthermore, we discuss the mechanism of clustering in ${ }^{8}$ Be which has the alpha-alpha cluster structure in the subsection 3.2. In these calculations, we adopt the bare nuclear interaction of the Argonne $v 8^{\prime}\left(\mathrm{AV}^{\prime}\right)$ (Wiringa \& Pieper, 2002) explained in the previous section and switch off the electromagnetic interactions.

\subsection{Description of the structures and energy-level schemes of light nuclei}

In Brueckner-AMD, the bound states of nucleus can be obtained as the solution of energy variation starting from the bare nuclear interactions without any assumption of configurations. However, in general, since the AMD wave functions are not good quantum states of the spin and parity $\left(J^{\pi}\right)$, one cannot calculate the energy-level scheme by a single AMD wave function and must project out the AMD wave functions onto the spin and parity eigenstates, which means the superposition of space-reflected and rotated Slater determinants (Kanada-En'yo et al., 1995). The parity projection means a linear combination of two Slater determinants:

$$
\left|\Phi^{ \pm}\right\rangle=\frac{1}{\sqrt{2}}(1 \pm \hat{P})|\Phi\rangle,
$$

where $\hat{P}$ is the space-reflection operator that operates at the spatial coordinate $\vec{Z}_{i}$ of each nucleon. In projecting out the wave functions onto each spin-parity $J^{ \pm}$state, the spin- and parity-projected state $\left|\Phi_{M K}^{J \pm}\right\rangle$ is expressed as

$$
\left|\Phi_{M K}^{J \pm}\right\rangle=\frac{2 J+1}{8 \pi^{2}} \int d \Omega D_{M K}^{J *}(\Omega) \hat{R}(\Omega)\left|\Phi^{ \pm}\right\rangle,
$$

where $D_{M K}^{J}(\Omega)$ is the Wigner $D$-function and $\hat{R}(\Omega)$ is the rotational operator that makes the spatial and spin coordinates rotated by the Euler angle $\Omega$. In the above equation, the integration means a linear combination of differential rotated Slater determinants. The energy expectation value of $\left|\Phi_{M K}^{J \pm}\right\rangle$ is given by

$$
\left\langle\hat{H}_{K^{\prime} K}^{J \pm}\right\rangle \equiv \frac{\left\langle\Phi_{M K^{\prime}}^{J \pm}|\hat{H}| \Phi_{M K}^{J \pm}\right\rangle}{\left\langle\Phi_{M K^{\prime}}^{J \pm} \mid \Phi_{M K}^{J \pm}\right\rangle}=\frac{\int d \Omega D_{K^{\prime} K}^{J *}(\Omega)\{\langle\Phi|\hat{R}(\Omega) \cdot \hat{H}| \Phi\rangle \pm\langle\Phi|\hat{P} \hat{R}(\Omega) \cdot \hat{H}| \Phi\rangle\}}{\int d \Omega D_{K^{\prime} K}^{J *}(\Omega)\{\langle\Phi|\hat{R}(\Omega)| \Phi\rangle \pm\langle\Phi|\hat{P} \hat{R}(\Omega)| \Phi\rangle\}} .
$$

In order to calculate the energy expectation value of the $J^{ \pm}$state correctly, we perform the $K$-mixing by diagonalizing the Hamiltonian matrix $\left\langle\Phi_{M K^{\prime}}^{J \pm}|\hat{H}| \Phi_{M K}^{J \pm}\right\rangle$ and norm matrix $\left\langle\Phi_{M K^{\prime}}^{J \pm} \mid \Phi_{M K}^{J \pm}\right\rangle$ simultaneously for the quantum numbers of $K$ and $K^{\prime}$. 

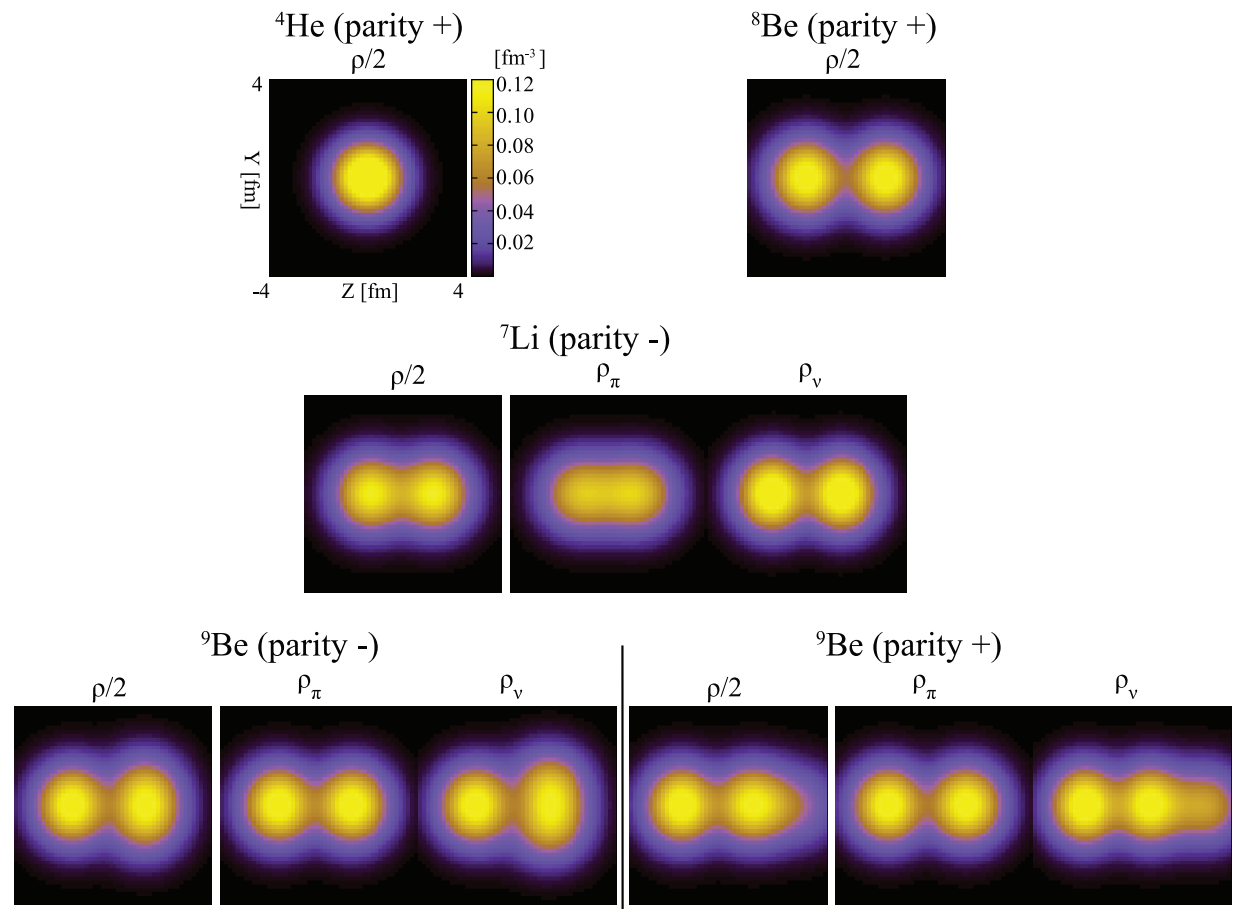

Fig. 4. Intrinsic matter $\rho$ densities as a half value, the proton $\rho_{\pi}$ and neutron $\rho_{v}$ ones of the lowest-energy parity solutions in ${ }^{4} \mathrm{He},{ }^{7} \mathrm{Li},{ }^{8} \mathrm{Be}$, and ${ }^{9} \mathrm{Be}$. The size of these squares is shown in the panel of ${ }^{4} \mathrm{He}$.

In these calculations, we determine the wave functions $|\Phi\rangle$ by performing the energy variation for the parity-projected states $\left|\Phi^{ \pm}\right\rangle$. Therefore, we can obtain not only the lowest-energy parity solution corresponding to the ground state but also the excited parity solution, whose parity is opposite to that of the lowest-energy state. Projecting out the solutions of the energy variation, $\left|\Phi^{ \pm}\right\rangle$, onto each $J$ state, we calculate the energy levels. In the following, we present the results of ${ }^{4} \mathrm{He},{ }^{7} \mathrm{Li},{ }^{8} \mathrm{Be}$, and ${ }^{9} \mathrm{Be}$ using the above procedure. As the Gaussian width parameter $v$ for every wave packet in Eq. (1), we adopt $v=0.222\left(\mathrm{fm}^{-2}\right)$ for ${ }^{4} \mathrm{He}$ and $v=0.208\left(\mathrm{fm}^{-2}\right)$ for other nuclei. Fig. 4 shows the intrinsic densities obtained from the wave functions $|\Phi\rangle$. For ${ }^{9} \mathrm{Be}$, both the lowest-energy negative-parity solution and the excited positive-parity one are shown. In these figures, the $X_{-}, Y_{-}$, and $Z$-axes in the body-fixed frame are chosen so as to be $\left\langle\sum_{i} z_{i}^{2}\right\rangle \geq\left\langle\sum_{i} y_{i}^{2}\right\rangle \geq\left\langle\sum_{i} x_{i}^{2}\right\rangle$, and each density distribution in the Z-Y plane is displayed. As seen in Fig. 4, the alpha particle $\left({ }^{4} \mathrm{He}\right)$ is compactly spherical and ${ }^{8} \mathrm{Be}$ consists of two-alpha cluster. In this result, the density of ${ }^{7} \mathrm{Li}$ does not present the explicit cluster structure as seen in an almost symmetric distribution. In the densities of ${ }^{9} \mathrm{Be}$, the structures of two-alpha cluster plus a valence neutron can be seen. In the negative-parity state of ${ }^{9} \mathrm{Be}$, the density distribution of the valence neutron corresponds to a $\pi$-molecular orbit; on the other hand, that of the positive-parity state corresponds to a $\sigma$-molecular orbit. 
In Table 1, the calculated binding energy of the ground state for each nucleus is listed to be compared with the experimental one. Although the calculated ground states are underbinding for the experimental ones, it is known that bare three nucleons interactions are needed to reproduce the experimental binding energies from accurate few-body calculations (Wiringa et al., 2000). However, in this work, there seems to be room for improvement to develop these calculations to the superposition with the wave functions having different configurations $\left\{\vec{Z}_{i}\right\}$ or Gaussian width parameters $v$. The calculated energy-level schemes of ${ }^{7} \mathrm{Li},{ }^{8} \mathrm{Be}$, and ${ }^{9} \mathrm{Be}$ are shown in Fig. 5. These energy levels are reproduced well. In the result of ${ }^{9} \mathrm{Be}$, the energy levels including the first excited states, $3 / 2_{2}^{-}, 5 / 2_{2}^{-}$, and $7 / 2_{2}^{-}$, which can be described by the $K$-mixing, are also reproduced well. In these calculations, in the negative-parity states of ${ }^{9} \mathrm{Be}$, the group of the dominant $K=3 / 2$ i.e. $K^{\pi}=3 / 2^{-}$band contains $3 / 2_{1}^{-}, 5 / 2_{1}^{-}$, and $7 / 2_{1}^{-}$states, while the $1 / 2_{1}^{-}, 3 / 2_{2}^{-}, 5 / 2_{2}^{-}$, and $7 / 2_{2}^{-}$states belong to the $K^{\pi}=1 / 2^{-}$band. The predicted $9 / 2_{1}^{-}$state is described coherently by the $K=1 / 2$ and $K=3 / 2$ states, which has almost equal weights to the $K=1 / 2$ and $K=3 / 2$. The positive-parity states of ${ }^{9} \mathrm{Be}$ are described as the $K^{\pi}=1 / 2^{+}$band.

\begin{tabular}{|c|c|c|c|}
\hline nucleus & $J^{\pi}$ & B-AMD $(\mathrm{MeV})$ & EXP $(\mathrm{MeV})$ \\
\hline${ }^{4} \mathrm{He}$ & $0^{+}$ & -24.6 & -28.3 \\
\hline${ }^{7} \mathrm{Li}$ & $3 / 2^{-}$ & -29.6 & -39.2 \\
\hline${ }^{8} \mathrm{Be}$ & $0^{+}$ & -44.0 & -56.5 \\
\hline${ }^{9} \mathrm{Be}$ & $3 / 2^{-}$ & -41.9 & -58.2 \\
\hline
\end{tabular}

Table 1. Total binding energies of the ground states for ${ }^{4} \mathrm{He},{ }^{7} \mathrm{Li},{ }^{8} \mathrm{Be}$, and ${ }^{9} \mathrm{Be}$. The column labeled " $J^{\pi}$ " lists the spin and parity of the ground state for each nucleus, and the columns labeled "B-AMD" and "EXP" list the calculated binding energies and the experimental ones, respectively.

\subsection{Investigation of the mechanism of alpha-alpha clustering}

In the following, we simulate the development of the alpha-alpha cluster in ${ }^{8} \mathrm{Be}$ and investigate its mechanism by using the bare nuclear interaction AV8 ${ }^{\prime}$ in the Brueckner-AMD framework. In this work, we construct the states that have the definite distance between two quasi-clusters consisting of four nucleons of the spin-up proton, spin-up neutron, spin-down proton, and spin-down neutron by using the constraint cooling method (Yamamoto et al., 2010). The definition of the distance between quasi-clusters in this case is represented as

$$
\left\langle d^{2}\right\rangle^{1 / 2}=\left|\vec{R}_{1}-\vec{R}_{2}\right|, \quad \vec{R}_{1}\left(\vec{R}_{2}\right)=\frac{1}{4} \sum_{i \in C_{1}\left(C_{2}\right)} \frac{\operatorname{Re} \vec{Z}_{i}}{\sqrt{v}},
$$

where the $\vec{R}_{1}\left(\vec{R}_{2}\right)$ represents the center of mass of the quasi-cluster $C_{1}\left(C_{2}\right)$ consisting of four nucleons. We take the $z$-axis as an inertia axis and the center of mass of quasi-clusters on the $z$-axis. We define a quasi-cluster as an initial set of nucleons that are chosen arbitrarily and determine a configuration of eight nucleons for ${ }^{8}$ Be by solving the following equation of the constraint cooling method as an energy variation:

$$
\frac{d \vec{Z}_{i}}{d t}=-\frac{\partial\langle H\rangle}{\partial \vec{Z}_{i}^{*}}-\frac{\partial C}{\partial \vec{Z}_{i}^{*}}, \quad C=C_{0} \cdot\left(\left\langle d^{2}\right\rangle^{1 / 2}-d\right)^{2},
$$



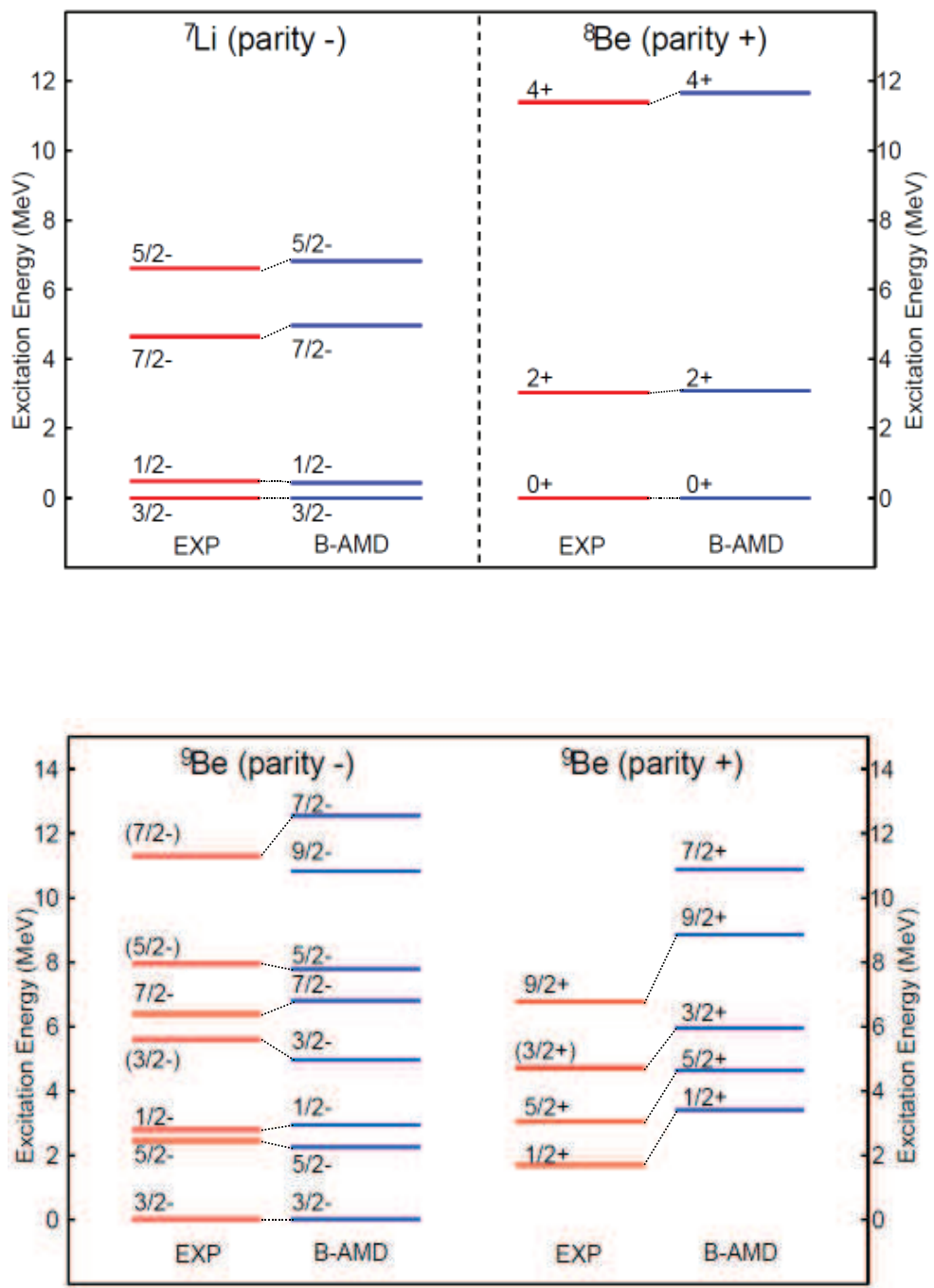

Fig. 5. Low-lying energy-level schemes of the experiments and Brueckner-AMD calculations labeled "EXP" and "B-AMD", respectively. The groud states are normalized to zero energy in these figures. Each energy level is represented as the line labeled its spin and parity. 


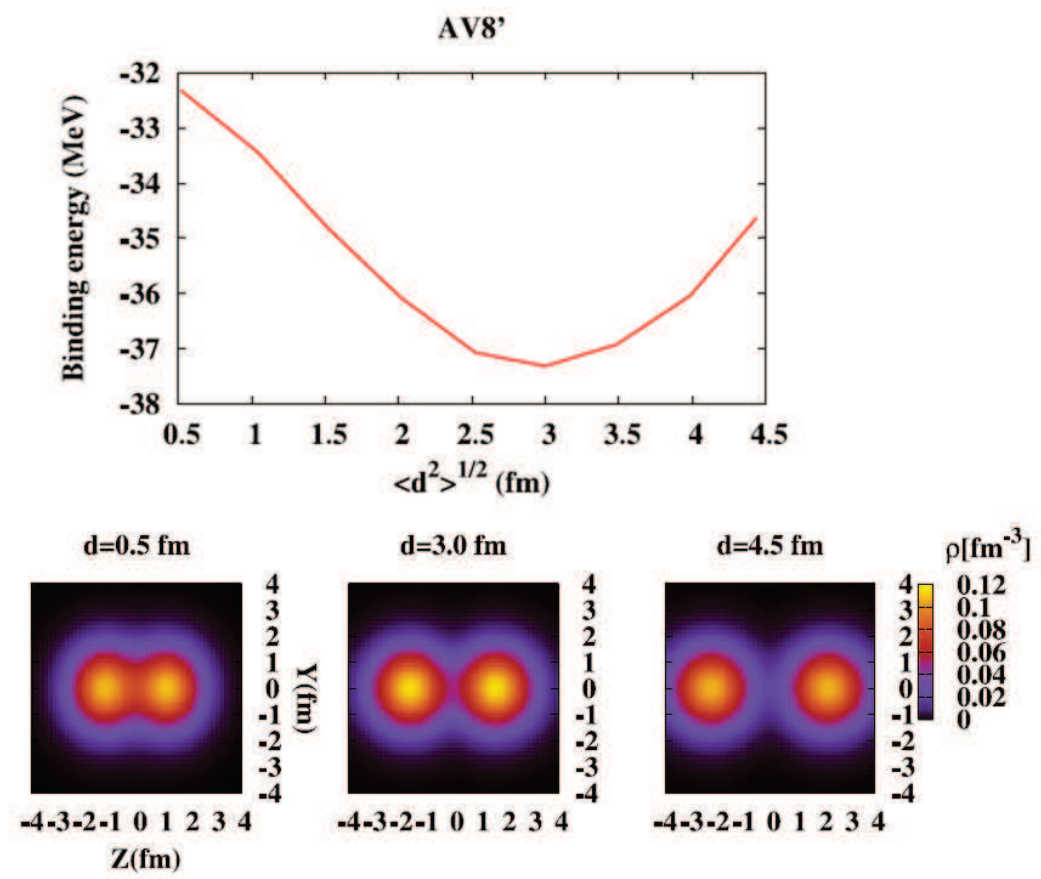

Fig. 6. Upper panel: Total binding energies of ${ }^{8} \mathrm{Be}$ are shown as a function of the distance of quasi-clusters. Lower panels: Density distributions of ${ }^{8} \mathrm{Be}$ at $d=0.5,3.0,4.5 \mathrm{fm}$.

where $C_{0}$ is the positive constant and $d$ is the constraint parameter; in this work, $d$ is the distance of quasi-clusters. The above equation makes it possible to obtain the solution of the energy variation under the constraint of a distance between two quasi-clusters. Note that the set of nucleons is not assumed to be localized spatially in this case. As a parameter of the wave function, we use the optimized Gaussian width for every wave packet in Eq. (1); $v=0.195\left(\mathrm{fm}^{-2}\right)$. We perform the energy variation and calculate the total binding energy for the parity-projected states corresponding to the lowest-energy parity solutions $\left|\Phi^{+}\right\rangle$in Eq. (56). In Fig. 6, the energy curve of calculated total binding energies are shown as a function of the distance between two quasi-clusters in the upper panel, and we show the intrinsic density distribution of wave functions obtained at different $d$-values $(d=0.5,3.0$, and $4.5 \mathrm{fm})$ in the lower panels. As seen in Fig. 6, one can see that the optimum distances are around $3.0 \mathrm{fm}$, where the alpha-alpha structure is realized starting from the bare nuclear interaction $\mathrm{AV} 8^{\prime}$, and the situation that the localized clusters are more developed at larger distances is reproduced as the results of the energy variation. In Fig. 7, we present single-particle energies and orbits at different distances $d$. Single-particle energies of eight nucleons are degenerated approximately into two types of energy $\varepsilon_{1}$ and $\varepsilon_{2}$ as seen in the upper panel of Fig. 7 . The two energy orbits correspond to gerade and ungerade orbits in the molecular orbital method (Bandō et al., 1970). However, in the present approach without the molecular-orbit assumption, the single-particle orbits describe not only molecular orbits but also atomic orbits of a mean field. At the small distance $d=0.5 \mathrm{fm}, \varepsilon_{2}-\varepsilon_{1}=15 \mathrm{MeV}$ are obtained in this calculation. That energy difference between the lowest $\left(\varepsilon_{1}\right)$ and the excited $\left(\varepsilon_{2}\right)$ single-particle 

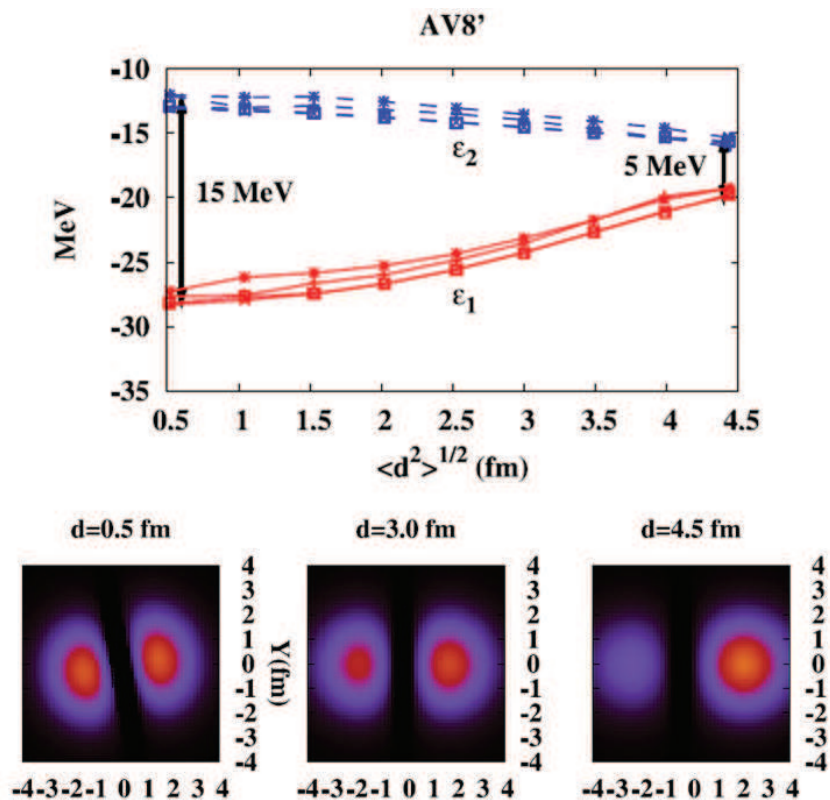

$\mathbf{Z}(\mathrm{fm})$
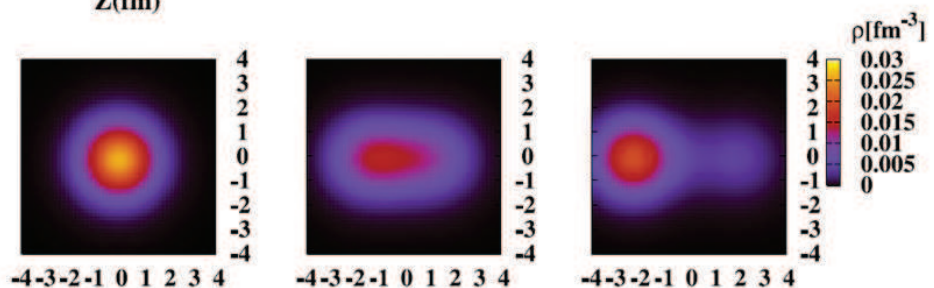

Fig. 7. Upper panel: Single-particle energies of ${ }^{8} \mathrm{Be}$ are shown. Lower panels: The density distributions of single-particle orbits for the two spin-up protons at $d=0.5,3.0,4.5 \mathrm{fm}$. The other single-particle orbits also have similar behavior. The upper and lower density distributions represent the higher-energy $\left(\varepsilon_{2}\right)$ and lower-energy $\left(\varepsilon_{1}\right)$ states, respectively.

orbits are consistent with $\hbar \omega$ of a shell model in nuclear systems. At the large distance $d=4.5$ $\mathrm{fm}, \varepsilon_{1}$ and $\varepsilon_{2}$ degenerate within $5 \mathrm{MeV}$. This result suggests that the single-particle orbits for $\varepsilon_{1}$ and $\varepsilon_{2}$ have configurations similar to each other. As shown in the lower panels of Fig. 7 , at the small distance region of $d=0.5 \mathrm{fm}$, the two types of single-particle energy $\varepsilon_{1}$ and $\varepsilon_{2}$ are understood as those of $s$-wave and $p$-wave orbits, respectively. The four nucleons of the $p$-wave orbit $\left(\varepsilon_{2}\right)$, which has a node at the origin, occupy around the $z$-direction in the intrinsic framework. This is the cause of the dumbbell shape of the density distribution at the small distance. On the other hand, at the large distance region far from $d=4.5 \mathrm{fm}$, these single-particle energies are expected to degenerate because all eight nucleons occupy the same type of single-particle orbit around different alpha clusters. At the intermediate distance region of $d=3.0 \mathrm{fm}$, they are interpreted to form gerade and ungerade orbits in the molecular orbital picture. In this work, we discuss the mechanism to cause this energy curve focusing on the state $(d)$ dependence of the G-matrix. For this purpose, we decompose the G-matrix 

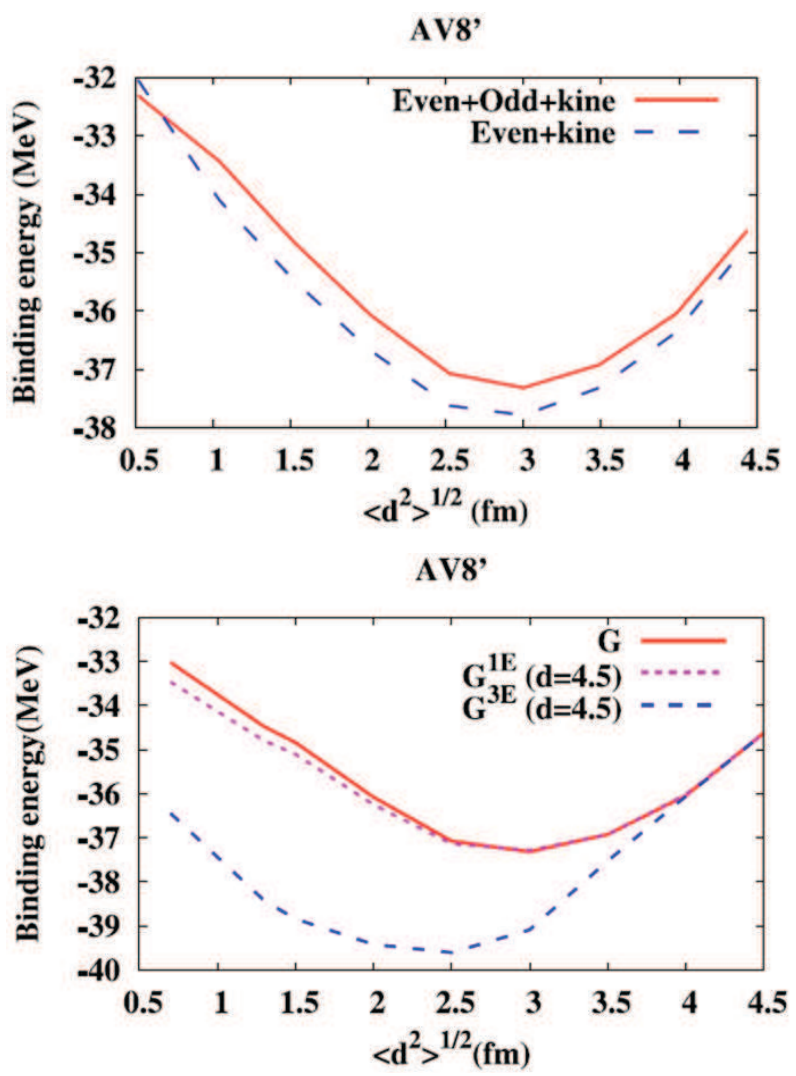

Fig. 8. Upper panel: Energy curves with and without odd-force contributions. The solid line, "Even+Odd+kine", and the dashed line, "Even+kine", represent the energy curves with and without the odd-force, respectively. Lower panel: the solid line, " $\mathrm{G}$ ", is the energy curve for the distance of quasi clusters, which is identical with the curve in the upper panel of Fig. 6, and the dotted line, " $\mathrm{G}^{1 E}(\mathrm{~d}=4.5)$ ", and dashed line, " $\mathrm{G}^{3 E}(\mathrm{~d}=4.5)$ ", are the energy curves calculated using the fixed $G$-matrices at $d=4.5 \mathrm{fm}$ for $1 \mathrm{E}$ and $3 \mathrm{E}$ channels, respectively.

into the two-body spin-parity channels and recalculate the energy curve fixing the G-matrix calculated at the largest $d=4.5(\mathrm{fm})$ for each two-body spin-parity channel. The upper panel of Fig. 8 indicates that the energy surface does not change even if the odd-force contributions of the triplet-odd $(3 \mathrm{O})$ and singlet-odd $(1 \mathrm{O})$ channels are taken off, and therefore, in the lower panel of Fig. 8, we focus on only the even-force contributions of the triplet-even (3E) and singlet-even $(1 \mathrm{E})$ channels. As seen in the lower panel of Fig. 8, the energy curve using the fixed $G$-matrix for the $1 \mathrm{E}$ channel has no difference from the original energy curve; the energy curve using the fixed G-matrix for the $3 \mathrm{E}$ channel is shifted largely from the original one. That means the 1E G-matrix has no state dependence but the 3E one is responsible for the state dependence of the G-matrix to cause the development of clusters in ${ }^{8} \mathrm{Be}$. This large state dependence is caused by the contributions of the tensor force of the bare nuclear interaction renormalized mainly by the correlation function $F_{k l}\left[\begin{array}{l}J=1 S=1 \\ l^{\prime}=2 l_{0}=0\end{array}\right]$ in Eq. (50). If the G-matrix had 
no state dependence, the energy curve would be deeper at a small distance, in other words, the actual energy curve becomes shallow due to the suppression of contributions at a small distance as the state dependence of the G-matrix and induces the separated clusters.

\section{Conclusion}

We develop a new ab initio framework, Brueckner-AMD, to makes AMD available with bare nuclear interactions. In this theory, we focus on the fact that one can apply the Brueckner theory straightforwardly to the AMD framework by using AMD-HF and the correlation functions constructed by the solutions of the Bethe-Goldstone equation make it possible to calculate the G-matrix in the AMD calculation. In these applications of Brueckner-AMD, we show that one can describe various states of nucleus starting from bare nuclear interactions with no assumption of a configuration. Furthermore, we simulate the development of alpha-alpha cluster in ${ }^{8} \mathrm{Be}$ and elucidate the origins of its formation in the Brueckner-AMD framework. The alpha-alpha clustering of ${ }^{8} \mathrm{Be}$ is induced by the contributions of the interaction for the triplet-even (3E) channel, where the 3E G-matrix has large state dependence caused by the correlations derived from the tensor force of the bare nuclear interaction.

\section{Acknowledgments}

We would like to express our gratitude to Professor K. Ikeda and Professor H. Horiuchi for their encouragements and useful comments. We also thank our collaborators, Mr. T. Murakami, Mr. Y. Yamamoto, Mr. T. Morimoto, and Professor M. Kimura in the Nuclear Theory Laboratory at Hokkaido University. This work is supported by the Grant-in-Aid for Exploratory Research.

\section{References}

Kamada, H., Nogga, A., Glöckle, W., Hiyama, E., Kamimura, M., Varga, K., Suzuki, Y., Viviani, M., Kievsky, A., Rosati, S., Carlson, J., Pieper, S. C., Wiringa, R. B., Navrátil, P., Barrett, B. R., Barnea, N., Leidemann, W., \& Orlandini, G. (2001). Benchmark test calculation of a four-nucleon bound state. Physical Review C, Vol.64, (August 2001), pp.044001, ISSN 0556-2813

Neff, T. \& Feldmeier, H. (2004). Cluster structures within Fermionic Molecular Dynamics. Nuclear Physics A, Vol.738, (June 2004), pp.357-361, ISSN 0375-9474

Wiringa, R. B.; Stoks, V. G. J. \& Schiavilla, R. (1995). Accurate nucleon-nucleon potential with charge-independence breaking. Physical Review C, Vol.51, No.1 (January 1995), pp.38-51, ISSN 0556-2813

Wiringa, R. B., Pieper, S. C., Carlson, J., \& Pandharipande, V. R. (2000). Quantum Monte Carlo calculations of $A=8$ nuclei. Physical Review C, Vol.62, (June 2000), pp.014001, ISSN 0556-2813

Wiringa, R. B.\& Pieper, S. C. (2002). Evolution of Nuclear Spectra with Nuclear Forces. Physical Review Letters, Vol.89, No.18, (October 2002), pp.182501, ISSN 0031-9007

Ikeda, K., Marumori, T., Tamagaki, R., \& Tanaka, H. (1972). Formation of the Viewpoint, Alpha-Like Four-Boby Correlations and Molecular Aspects in Nuclei. Progress Theoretical Physics Supplement, No.52, (December 1972), pp.1-24, ISSN 1347-0481 
Kanada-En'yo, Y.; Horiuchi, H. \& Ono, A. (1995). Structure of Li and Be isotopes studied with antisymmetrized molecular dynamics. Physical Review C, Vol.52, No.2, (August 1995), pp.628-646, ISSN 0556-2813

Togashi, T. \& Katō, K. (2007). Brueckner-AMD Method and Its Applications to Light Nuclei. Progress Theoretical Physics, Vol.117, No.1, (January 2007), pp.189-194, ISSN 1347-0481

Togashi, T.; Murakami, T. \& Katō, K. (2009). Description of Nuclear Structures with Brueckner-AMD plus $J^{\pi}$ Projection. Progress Theoretical Physics, Vol.121, No.2, (February 2009), pp.299-317, ISSN 1347-0481

Doté, A.; Horiuchi , H. \& Kanada-En'yo, Y. (1997). Antisymmetrized molecular dynamics plus Hartree-Fock model and its application to Be isotopes. Physical Review C, Vol.56, No.4, (October 1997), pp.1844-1854, ISSN 0556-2813

Myo, T.; Toki, H. \& Ikeda, K. (2009). Tensor-Optimized Shell Model with Bare Nucleon-Nucleon Interaction for ${ }^{4}$ He. Progress Theoretical Physics, Vol.121, No.3, (March 2009), pp.511-531, ISSN 1347-0481

Baldo, M., Fiasconaro, A., Song, H. Q., Giansiracusa, G., \& Lombardo, U. (2001). High density symmetric nuclear matter in the Bethe-Brueckner-Goldstone approach. Physical Review C, Vol.65, (December 2001), pp.017303, ISSN 0556-2813

Bandō, H.; Nagata, S. \& Yamamoto, Y. (1970). Reaction Matrix Theory for Cluster States in Light Nuclei. I -Be ${ }^{8}$-. Progress Theoretical Physics, Vol.44, No.3, (September 1970), pp.646-662, ISSN 1347-0481

Yamamoto, Y.; Togashi, T. \& Katō, K. (2010). Tensor Force Effects on $\alpha-\alpha$ Clustering of ${ }^{8}$ Be. Progress Theoretical Physics, Vol.124, No.2, (August 2010), pp.315-330, ISSN 1347-0481 


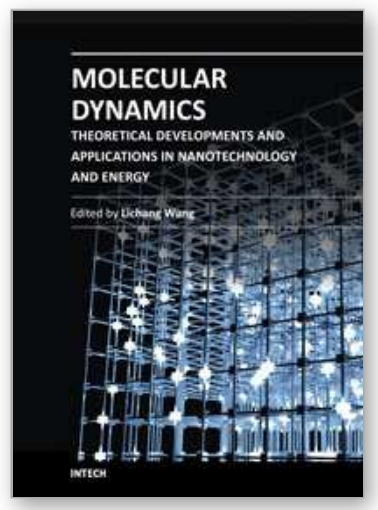

\author{
Molecular Dynamics - Theoretical Developments and Applications \\ in Nanotechnology and Energy \\ Edited by Prof. Lichang Wang
}

ISBN 978-953-51-0443-8

Hard cover, 424 pages

Publisher InTech

Published online 05, April, 2012

Published in print edition April, 2012

Molecular Dynamics is a two-volume compendium of the ever-growing applications of molecular dynamics simulations to solve a wider range of scientific and engineering challenges. The contents illustrate the rapid progress on molecular dynamics simulations in many fields of science and technology, such as nanotechnology, energy research, and biology, due to the advances of new dynamics theories and the extraordinary power of today's computers. This first book begins with a general description of underlying theories of molecular dynamics simulations and provides extensive coverage of molecular dynamics simulations in nanotechnology and energy. Coverage of this book includes: Recent advances of molecular dynamics theory Formation and evolution of nanoparticles of up to 106 atoms Diffusion and dissociation of gas and liquid molecules on silicon, metal, or metal organic frameworks Conductivity of ionic species in solid oxides Ion solvation in liquid mixtures Nuclear structures

\title{
How to reference
}

In order to correctly reference this scholarly work, feel free to copy and paste the following:

Tomoaki Togashi and Kiyoshi Katō (2012). Antisymmetrized Molecular Dynamics with Bare Nuclear Interactions: Brueckner-AMD, and Its Applications to Light Nuclei, Molecular Dynamics - Theoretical Developments and Applications in Nanotechnology and Energy, Prof. Lichang Wang (Ed.), ISBN: 978-953-510443-8, InTech, Available from: http://www.intechopen.com/books/molecular-dynamics-theoreticaldevelopments-and-applications-in-nanotechnology-and-energy/antisymmetrized-molecular-dynamics-withbare-nuclear-interactions-brueckner-amd-and-its-applications

\section{INTECH}

open science | open minds

\section{InTech Europe}

University Campus STeP Ri

Slavka Krautzeka 83/A

51000 Rijeka, Croatia

Phone: +385 (51) 770447

Fax: +385 (51) 686166

www.intechopen.com

\section{InTech China}

Unit 405, Office Block, Hotel Equatorial Shanghai

No.65, Yan An Road (West), Shanghai, 200040, China 中国上海市延安西路65号上海国际贵都大饭店办公楼405单元

Phone: +86-21-62489820

Fax: +86-21-62489821 
(C) 2012 The Author(s). Licensee IntechOpen. This is an open access article distributed under the terms of the Creative Commons Attribution 3.0 License, which permits unrestricted use, distribution, and reproduction in any medium, provided the original work is properly cited. 